\title{
Developmental Trajectories of Acculturation: Links With Family Functioning and Mental Health in Recent-Immigrant Hispanic Adolescents
}

\author{
Seth J. Schwartz \\ University of Miami \\ Byron L. Zamboanga \\ Smith College \\ Craig A. Mason \\ University of Maine \\ Lourdes Baezconde-Garbanati \\ University of Southern California
Sabrina E. Des Rosiers
Barry University \\ Juan A. Villamar \\ Northwestern University
}

\author{
Jennifer B. Unger \\ University of Southern California \\ David Córdova \\ University of Michigan \\ Shi Huang \\ University of Miami \\ Elma I. Lorenzo-Blanco \\ University of South Carolina \\ Daniel W. Soto \\ University of Southern California \\ Monica Pattarroyo \\ University of Southern California
}

Karina M. Lizzi and José Szapocznik
University of Miami

\begin{abstract}
The present study was designed to examine acculturative changes, and their effects on mental health and family functioning, in recent-immigrant Hispanic adolescents. A sample of 302 Hispanic adolescents was assessed five times over a $2 \frac{1}{2}-$-year period. Participants completed measures of Hispanic and U.S. practices, collectivist and individualist values, and ethnic and U.S. identity at each time point. Baseline and Time 5 levels of mental health and family functioning were also assessed. Latent class growth analyses produced two-class solutions for practices, values, and identifications. Adolescents who increased over time in practices and values reported the most adaptive mental health and family functioning. Adolescents who did not change in any acculturation domain reported the least favorable mental health and family functioning.
\end{abstract}

\footnotetext{
Preparation of this article was supported by Grants DA026594 from the National Institute on Drug Abuse and AA021888 from the National Institute on Alcohol Abuse and Alcoholism to Seth J. Schwartz, and by National Center for Advancing Translational Sciences Grant 1UL1TR000460 to José Szapocznik. We thank Maria-Rosa Velazquez, Tatiana Clavijo, Mercedes Prado, Alba Alfonso, Aleyda Marcos, Daisy Ramirez, Lissette Ramirez, and Perlita Carrillo for their hard work conducting assessments and tracking families; Dr. Judy Arroyo for her guidance and wisdom; and the study families for sharing their experiences with us.

Correspondence concerning this article should be addressed to Seth J. Schwartz, Department of Public Health Sciences, Leonard M. Miller School of Medicine, University of Miami, Miami, FL 33136. Electronic mail may be sent to sschwartz@med.miami. edu.
}

Rates of international migration have increased steadily since the middle of the 20th century and are now the highest in modern history (Steiner, 2009). For example, more than 40 million foreignborn people reside in the United States (Grieco et al., 2012). Additionally, over 14 million of these immigrants arrived after 2000, 7 million after 2005, and 3 million after 2008 (Walters \& Trevelyan,

(C) 2015 The Authors

Child Development (c) 2015 Society for Research in Child Development, Inc. All rights reserved. 0009-3920/2015/8603-0006

DOI: $10.1111 /$ cdev. 12341 
2011). The U.S. Census Bureau predicts that immigration will represent the primary source of population growth at least through 2050 (Bernstein, 2013) -meaning that issues related to immigration will continue to be important to examine.

Since 1965, when restrictive U.S. immigration laws were changed, the majority of immigrants entering the United States have been from Latin America, Asia, and the Caribbean (Grieco et al., 2012). Indeed, the top 10 sending countries for immigrants arriving since 1980 include Mexico, Cuba, the Dominican Republic, and El Salvador (Walters \& Trevelyan, 2011). Not surprisingly, Hispanics have now become the largest and fastest growing ethnic minority group in the United States, representing approximately $17 \%$ of all legal U.S. residents (Ennis, Rios-Vargas, \& Albert, 2011). Other than Puerto Ricans, who are U.S. citizens at birth, the two largest Hispanic groups in the United States are Mexicans and Cubans. Los Angeles and Miami, respectively, have traditionally been the two largest cities of settlement for these two groups (Hayes-Bautista, 2004; Stepick, Grenier, Castro, \& Dunn, 2003).

Hispanics are an important group to study not only because of their size and growth rate but also because of the sociopolitical context that surrounds their immigration to the United States. Hispanic immigration has been a "lightning rod" for pushback from some White Americans for two general reasons: (a) Hispanics comprise the majority of undocumented immigrants in the United States (Pew Hispanic Center, 2010) and (b) the vast majority of Hispanics share Spanish as a common language and often maintain Spanish into the second and even third generations. Social commentators such as Huntington (2004) have expressed concerns regarding the effects of undocumented immigration on the U.S. economy and regarding the effects of the continuing spread of Spanish on the status of English as the national language of the United States. These concerns have created an ambivalent context of reception for many Hispanic immigrants: They are welcomed by large coethnic communities in many U.S. cities but are viewed as a threat by many White Americans (e.g., Henderson, 2011). This ambivalent context of reception undoubtedly shapes the ways in which Hispanic immigrants adapt following their arrival in the United States.

\section{Acculturation}

For most immigrants, a process of acculturation occurs following arrival in the society of settlement.
As applied to international migration, acculturation refers to a process of cultural change where the person adopts some (or all) aspects of the new country's cultural streams and retains some (or all) aspects of the heritage country's cultural streams (Sam \& Berry, 2010). Acculturation is inherently a developmental process, where receiving-culture acquisition and heritage-culture retention increase, remain stable, or decrease over time (Berry, 1997). Accordingly, research has begun to progress from one-time, cross-sectional assessments of acculturation to more dynamic, longitudinal investigations of acculturation over time (e.g., Knight et al., 2009).

Although acculturation has been studied in a variety of populations and age groups, studying developmental trajectories of acculturation might best be done with adolescents and young adults who are undergoing an acute cultural transformation-and who are likely to evidence the greatest degree of acculturation. Cheung, Chudek, and Heine (2011) have identified a "sensitive period" for acculturation-adolescents and young adults who had resided in the country of settlement for a relatively small number of years. A recent-immigrant adolescent sample may therefore be ideal for studying developmental trajectories of acculturation.

In order for longitudinal work on acculturation to be most fruitful, it must be based on comprehensive models of acculturation that reflect the lived experiences of immigrants. Furthermore, not only is it essential to assess acculturation, but it is also important to assess its effects on quality-of-life indices among immigrants. For example, variables related to acculturation may be positively (UmañaTaylor, Gonzales-Backen, \& Guimond, 2009) or negatively (Forster, Grigsby, Soto, Schwartz, \& Unger, 2014) predictive of mental health outcomes, depending on the specific acculturation-related variable and mental health outcome under consideration. Acculturation can also exert its influence on family relationships as adolescents adopt U.S. cultural orientations and may begin to drift away from their heritage cultural orientations (Unger, Ritt-Olson, Soto, \& Baezconde-Garbanati, 2009). Our study therefore examined both (a) developmental trajectories of acculturation and (b) the effects of these trajectories on mental health and family functioning outcomes. We review each of these issues in the next two sections-first, what acculturation is and how it might change over time, and second, how acculturation might be related to mental health and family functioning outcomes. 


\section{The Role of Acculturation in Adolescent Development}

As the populations of many countries have continued to diversify, cultural concerns have become important within many lines of research. Developmental science, in particular, is grounded in interactions between the individual and her or his social and cultural context (García Coll, Akerman, \& Cicchetti, 2000; Lerner \& Galambos, 1998). Cultural processes such as acculturation may exert important influences on developmental outcomes - especially in adolescence when young people are beginning to consider who and what they are and wish to become (Cooper, 2011). Adolescents from immigrant and minority backgrounds may be especially challenged with culturally related identity issues. Specifically, they must find their place within their cultures of origin and within the larger society in which they reside - a challenge that may involve attempting to reconcile seemingly incompatible roles and expectations (e.g., Tropp, Erkut, García Coll, Alarcón, \& Vázquez García, 1999). Maintaining harmonious relationships with traditionally oriented family members, as well as manifesting positive mental health outcomes, may depend on the adolescent's ability to live within two cultures. Indeed, living within two cultures is a major component of the acculturation process.

\section{Models of Acculturation}

The conceptualization and measurement of acculturation have evolved considerably over the years. Early acculturation models and measures were unidimensional, in that adopting the receiving culture was equated with renouncing one's heritage culture (Gordon, 1964). However, such a model is not consistent with the lived reality of immigrants. Principally, the majority of young immigrants are bicultural, highly endorsing both U.S. culture and their cultures of origin (Nguyen \& Benet-Martínez, 2013). Unlike unidimensional acculturation models, bidimensional models recognize biculturalism (strong endorsement of both U.S. and heritage cultural streams) as the most common, and often the most adaptive, acculturation approach-especially for young immigrants (Nguyen \& Benet-Martínez, 2013).

Acculturation is multidimensional not only in terms of the cultural streams (e.g., Hispanic, United States) that are included under its auspices but also in terms of the content domains that it encompasses (LaFromboise, Coleman, \& Gerton, 1993). Each cultural dimension (e.g., Hispanic culture retention,
U.S. culture acquisition) can be measured in at least three domains - practices, values, and identifications (Schwartz, Unger, Zamboanga, \& Szapocznik, 2010). Practices refer to behaviors such as language use, culinary preferences, and choice of friends. Values refer to how individuals prioritize their own needs vis-à-vis those of their families and communities. Specifically, individualistic values, often associated with the United States (Hirschman, 2003), refer to an emphasis placed on competition and individual achievement (Knight et al., 2010) — whereas collectivist values, often associated with Hispanic cultural contexts, refer to a focus on family obligations, family honor, and respect for elders (Triandis, 1995). Identifications refer to the extent to which one feels attached to one's heritage-cultural group, country, or region, and/or to the United States (i.e., ethnic identity and national identity). Broadly, practices represent a behavioral domain of acculturation, values represent a cognitive domain of acculturation, and identifications represent an affective domain of acculturation (Castillo \& Caver, 2009). Figure 1 illustrates this model, where dimensions (United States or Hispanic) are plotted on the $x$-axis, domains are plotted on the $y$-axis, and each dimension-domain pairing represents an acculturation component. The model therefore consists of six acculturation components.

Practices, values, and identifications are correlated with, but still at least somewhat independent from, one another. For example, although individuals who are highly ethnically identified are often at least somewhat fluent in their heritage language, Portes and Rumbaut (2006) provide numerous examples of Hispanic and Asian immigrant adolescents who have lost proficiency in their native

\section{DOMAIN DIMENSION}

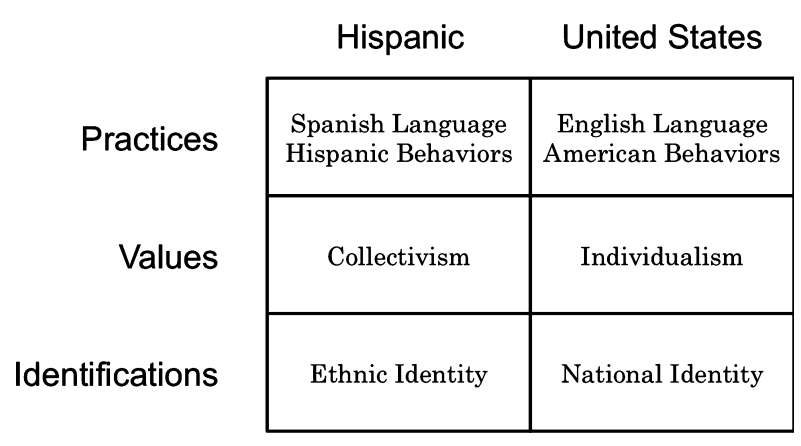

Figure 1. Our theoretical model is described as the intersection of cultural dimensions (United States and Hispanic) and domains (practices, values, and identifications). Each cell in this figure represents an acculturation component. 
languages but who nonetheless strongly identify with their ethnic heritage. Identifying with one's heritage does not necessarily mean that one will hold the values of, or engage in the practices characteristic of, that heritage group.

Empirical tests of our bidimensional, multidomain acculturation model suggest that within each dimension (receiving culture acquisition or heritage culture retention), practices, values, and identifications are modestly intercorrelated at one point in time (Schwartz et al., 2011). Longitudinal studies have separately mapped developmental trajectories of cultural practices (Knight et al., 2009), cultural values (Knight et al., 2013), and cultural identifications (Knight et al., 2009) among Hispanic adolescents. What remains to be done, however, is to examine the overlap between trajectories of the various acculturation components. The fact that these components are interrelated at one point in time does not provide information about the extent to which they would change in tandem. Put differently, the fact that someone appears to be bicultural in terms of cultural practices does not provide information about how that person might score in terms of cultural values or identifications, or whether this biculturalism would also emerge as an over-time trajectory.

\section{Acculturation, Family Functioning, and Mental Health}

Much of the acculturation literature has focused not only on ascertaining the structure of acculturation and examining patterns among different groups of immigrants but also on investigating the links between acculturative processes and mental health indices. Most of these studies, however, have used cross-sectional or short-term longitudinal designs, with very few studies examine acculturation trajectories as predictors of mental health. Mental health outcomes that have been associated with acculturation in cross-sectional and short-term longitudinal studies include self-esteem (Smokowski \& Bacallao, 2007), internalizing symptoms (Smokowski, Rose, \& Bacallao, 2010), and prosocial behavior (behaving positively toward, and helping, others; Schwartz, Zamboanga, \& Jarvis, 2007) - where prosocial behavior might be considered as an indicator of mental health because it is an indicator of positive development among adolescents (Carlo \& Randall, 2002). Generally speaking, among Hispanic immigrant adolescents, retention of one's cultural heritage is positively associated with self-esteem and prosocial behavior, and negatively associated with internalizing symptoms. The effects of acquir- ing U.S. culture are less clear, with some studies finding U.S. culture acquisition to be associated with problematic outcomes (González-Wahl \& McNulty-Eitle, 2010) but others not finding such an association (Schwartz et al., 2011). Biculturalismwhich involves both retaining one's cultural heritage and acquiring the receiving culture-is even more facilitative of positive mental health and protective against internalizing symptoms (Nguyen \& Benet-Martínez, 2013). Biculturalism appears to be protective because it allows the person to select behaviors from either cultural stream to match the cultural context associated with various situations (LaFromboise et al., 1993). Again, however, much of the research examining links between biculturalism and mental health has been cross-sectional.

A number of studies have also examined family functioning as a correlate of acculturation among adolescents from immigrant families. These studies can be divided into at least two general categories: (a) those that have examined the direct effects of adolescent acculturation on parent-adolescent involvement, communication, conflict, or other family processes (e.g., Gonzales, Deardorff, Formoso, Barr, \& Barrera, 2006), and (b) those that have examined the effects of parent-adolescent acculturation discrepancies on parent-adolescent involvement, communication, conflict, or other family processes (e.g., Lau et al., 2005; Unger et al., 2009). In the majority of cases, either higher adolescent U.S. acculturation or greater discrepancies in U.S. acculturation between adolescents and parents (which generally reflect higher adolescent acculturation to the United States) predicted compromised parent-adolescent relationships (e.g., greater conflict, lower cohesion, poorer communication). Heritage-culture retention, on the other hand, has been associated with more favorable family processes (e.g., Schwartz, Unger, et al., 2012).

Again, however, the majority of studies linking acculturation to mental health or family functioning have been cross-sectional or short-term longitudinal. Even those studies using longitudinal designs have generally analyzed acculturative processes only at one time point-meaning that the links of acculturation trajectories with mental health and family functioning have been largely unexplored. A notable exception is Schwartz et al. (2013), who examined family functioning as a mediator of the effect of trajectories of Hispanic and U.S. cultural practices on adolescent substance use and sexual behavior.

No published studies (at least to our knowledge), however, have simultaneously (a) examined trajec- 
tories of cultural practices, values, and identifications; (b) ascertained the overlap among trajectories for these three acculturation domains; and (c) mapped the effects of these combined trajectories on indices of mental health and family functioning. Some empirical research has hinted at such a difference in patterns. Knight and colleagues (Knight et al., 2009; Knight et al., 2013) examined developmental trajectories of cultural practices and Hispanic ethnic identity in a sample of juvenile offenders and examined developmental trajectories of cultural values in a community sample of Mexican American youth. In the juvenile offender sample, Knight et al. (2009) found two trajectory classes for language use, two classes for peer affiliations, and two classes for ethnic identity - but these solutions bore little resemblance to each other. In the community sample, Knight et al. (2013) found four trajectory classes, some of which resembled language use, peer affiliation, or ethnic identity classes from the juvenile offender study (e.g., increasing U.S. values and decreasing Mexican values). It should be noted, however, that the majority of adolescents in both of these samples were U.S.-born.

\section{The Present Study}

In the present study, we extended Knight and colleagues (Knight et al., 2009; Knight et al., 2013) and others by examining developmental trajectories in the acculturation components included in our theoretical model-Hispanic and U.S. practices, values, and identifications. Following Knight et al. (2010), we examined U.S. and Hispanic components jointly within each domain - that is, we empirically extracted separate class solutions for Hispanic and U.S. practices, for collectivist and individualist values, and for ethnic and U.S. identity. We then classified each participant into her or his most likely class and cross-tabulated these three sets of classes to create a superordinate set of categories (e.g., Class 1 for practices, Class 2 for values, and Class 1 for identifications). Next, we compared each of the outcome variables across these superordinate categories. Finally, we estimated separate latent class models for each acculturation domain, rather than estimating a single latent class model for all six acculturation components, to maintain the conceptual and empirical independence among the three domains.

We conducted our study in both Los Angeles and Miami, so that we would be able to capture more diversity in the U.S. Hispanic population than would be available in either city alone. These cities were selected because they are the most common destinations for the two largest Hispanic immigrant groups in the United States (Mexicans and Cubans, respectively). A multisite design was used to expand on prior longitudinal trajectory modeling on acculturation, which has been conducted in single locations (e.g., Knight et al., 2009, conducted their study in Phoenix; Schwartz et al., 2013, conducted their study in Miami). Studying acculturation, and its effects on mental health and family functioning, among adolescents in these two cities simultaneously might therefore provide a more accurate portrayal of acculturation and its effects on mental health and family functioning than would studying only one location.

Our two-site study provides two cities with large Hispanic populations and in which Hispanics are received quite differently. Cuban immigrants transformed Miami from a port and vacation destination into a major city, and Hispanics continue to hold the majority of economic and political power in the city (Stepick et al., 2003). On the other hand, although Los Angeles is home to the largest Mexican-descent population in the United States, the context of reception for Hispanics in Los Angeles might best be characterized as ambivalent (Light, 2006). For example, although former Los Angeles mayor Antonio Villaraigosa is Mexican American, use of Spanish in public places is less widely encouraged than it is in Miami. Attachment to the society of settlement tends to be lower, and attachment to the heritage cultural group tends to be higher, in less welcoming contexts of reception (Rumbaut, 2008). As a result, we hypothesized smaller increases (or greater decreases) in U.S. practices, values, and identifications over time-and larger increases (or smaller decreases) in Hispanic practices, values, and identifications over time - in Los Angeles than in Miami.

We also hypothesized that multiple classes would emerge for each of the three acculturation domains, but we did not advance specific a priori hypotheses regarding the content of these classes. Given that the adolescents in our sample were recently immigrated (i.e., resided in the United States for 5 years or less at baseline), we anticipated that Hispanic orientations would be highly endorsed across time in all of the classes extracted. Regarding the links of acculturation trajectory classes with indices of mental health and family functioning, we hypothesized that classes characterized by biculturalism-high and/or increasing endorsement of Hispanic and U.S. practices, values, and identifications across time-would be linked with the most favorable mental health and family 
functioning scores at the final time point. Given that biculturalism is associated with the most favorable mental health outcomes (Nguyen \& BenetMartínez, 2013), classes where the youth was not moving toward biculturalism (i.e., low or decreasing endorsement of either Hispanic or U.S. cultural streams) in all of the three domains would be expected to be associated with lower scores on mental health and family functioning, and classes where the person was not bicultural in any of the three domains would be expected to be associated with the lowest scores on mental health and family functioning.

\section{Method \\ Participants}

The sample for the present study consisted of 302 recent-immigrant Hispanic adolescents (53\% boys; $M_{\text {age }}=14.51$ years at baseline, $S D=0.88$ years, range $=14-17$ ). As per inclusion criteria, all adolescents had arrived in the United States within 5 years of the baseline data collection and were either finishing or entering the ninth grade. Adolescents in Miami $(n=152)$ had been in the United States for a median of 1 year (interquartile range $[\mathrm{IQR}]=0$ 3 years), whereas adolescents in Los Angeles $(n=150)$ had been in the United States for a median of 3 years (IQR $=1-4$ years).

The Miami sample was primarily from Cuba (61\%), the Dominican Republic (8\%), Nicaragua
$(7 \%)$, Honduras $(6 \%)$, Colombia $(6 \%)$, and other Hispanic countries (12\%), and the Los Angeles sample was primarily from Mexico (70\%), El Salvador (9\%), Guatemala (6\%), and other Hispanic countries $(15 \%)$. The mean annual household income, as reported by parents, was $\$ 30,854(S D=\$ 10,824)$. Demographic differences between the Miami and Los Angeles samples are reported in Table 1.

\section{Procedures}

Baseline data were gathered during the summer and fall of 2010, and subsequent time points occurred during spring 2011, fall 2011, spring 2012, and fall 2012. Participants were recruited from randomly selected public schools in heavily Hispanic areas in Miami-Dade and Los Angeles counties. Because (a) we were interested in recent-immigrant families and (b) many Hispanic immigrants tend to settle in heavily Hispanic areas (Kasinitz, Mollenkopf, Waters, \& Holdaway, 2008; Stepick et al., 2003), we selected schools where the student body was at least $75 \%$ Hispanic. Our goal was to recruit 25 students per school for a total of 150 families per site. In cases where a school or district did not provide at least 25 students, we recruited additional students from another nearby high school. The study was approved by the Institutional Review Boards at the University of Miami and the University of Southern California, and by the Research Review Committees for each of the participating school districts.

Table 1

Demographic Differences Between the Miami and Los Angeles Subsamples

\begin{tabular}{|c|c|c|c|}
\hline Variable & Miami & Los Angeles & Statistical test \\
\hline Countries of origin & $\begin{array}{l}61 \% \text { Cuban } \\
8 \% \text { Dominican } \\
7 \% \text { Nicaraguan } \\
6 \% \text { Honduran } \\
6 \% \text { Colombian } \\
4 \% \text { Salvadoran } \\
3 \% \text { Mexican } \\
2 \% \text { Peruvian } \\
3 \% \text { other countries }\end{array}$ & $\begin{array}{l}70 \% \text { Mexican } \\
9 \% \text { Salvadoran } \\
6 \% \text { Guatemalan } \\
4 \% \text { Honduran } \\
3 \% \text { Nicaraguan } \\
3 \% \text { Peruvian } \\
5 \% \text { other countries }\end{array}$ & $\mathrm{N} / \mathrm{A}$ \\
\hline Percentage of families arriving together & $83 \%$ & $67 \%$ & $\chi^{2}(1)=9.76^{* *}, \phi=.19$ \\
\hline Duration of separation (years) ${ }^{\mathrm{a}}$ & $2.72(1.65)$ & $2.28(1.67)$ & $t(66)=1.06, d=0.28$ \\
\hline Years in the U.S. ${ }^{\mathrm{b}}$ & $\mathrm{M} d n=1, \mathrm{IQR}=0-3$ & $\mathrm{M} d n=3, \mathrm{IQR}=1-4$ & Wilcoxon $z=6.39^{* * *}$ \\
\hline Annual family income $\mathrm{b}^{\mathrm{b}}$ & $\$ 27,028(S D=\$ 13,454)$ & $\$ 34,521(S D=\$ 5,398)$ & $t(178)=6.09^{* * *}$ \\
\hline Years of school completed (parent) ${ }^{\mathrm{b}}$ & $11.17(3.72)$ & $8.84(4.47)$ & $t(289)=4.92^{* * *}$ \\
\hline Parent employed past year ${ }^{\mathrm{b}}$ & $39.9 \%$ & $52.7 \%$ & $\chi^{2}(1)=4.99^{*}$ \\
\hline
\end{tabular}

${ }^{\mathrm{a}}$ Includes only families who reported an immigration-related separation. ${ }^{\mathrm{b}}$ At baseline. $* p<.05 . * *<.01 . * * * p .001$. 
At each school, we first obtained approval from the principal or vice-principal to conduct the study. In Miami, because the majority of new Hispanic immigrant students are enrolled in English for Speakers of Other Languages (ESOL) classes, we gave a brief presentation in each ESOL class about the study and asked interested students to provide their primary caregiver's phone number. We also gave presentations in the basic-level English classes into which students would transition after completing the ESOL program. In Los Angeles, we also approached students in ESOL classes-but because students in California are transferred out of ESOL after 1 year, we also recruited from the student body at large. In some schools in Los Angeles, principals were able to supply us with a list of students who had been in the United States for 5 years or less. All participating schools were public high schools. In Miami, 10 schools participated, and the number of participating students within each school ranged from 1 to $57(M d n=9, I Q R=4-19)$. In Los Angeles, 13 schools participated, and the number of students participating from each school ranged from 1 to $27(M d n=12, \mathrm{IQR}=4-16)$.

Staff members called parents to verify that the adolescent had been in the United States for less than 5 years and that the family planned to remain in the South Florida or Southern California area during the course of the study. Parents whose adolescents met these inclusion criteria were invited to schedule evening or weekend assessment appointments at a convenient location. We received contact information for 632 adolescents who met the study's inclusion criteria. Of these, 197 were unreachable, primarily because of incorrect or nonworking telephone numbers. The remaining 435 families were reached by telephone and invited to participate. Of these 435 families, 69\% $(n=302)$ participated in the study. A total of 133 families met the inclusion criteria and were contacted, but did not participate, 93 (65\%) of these families were unable to participate due to reported work or scheduling conflicts, $18(13 \%)$ missed at least three scheduled assessment appointments, $1(1 \%)$ was planning to move, 2 ( $2 \%)$ reported experiencing serious health problems, and $19(14 \%)$ declined but did not provide a reason. Adolescents received a movie ticket for each time point at which they participated.

Parents and adolescents provided informed consent and assent, respectively. Parents and adolescents were taken to separate rooms so that the consent/assent process could be conducted privately. In cases where adolescents declined to provide assent, parents were told that the family did not meet the inclusion criteria (to protect the adolescent's privacy). For the present study, only adolescent-report measures were used in the analysis.

Each participant completed the assessment battery in English or Spanish, according to her or his preference. Eighty-four percent of adolescents completed their assessments in Spanish at baseline. This percentage decreased to $77 \%$ at Time $2,72 \%$ at Time $3,66 \%$ at Time 4 , and $68 \%$ at Time 5. Empirical evidence (Schwartz, Benet-Martínez, et al., 2014) suggests that it may be safe to pool acculturation data across languages of assessment. Assessments were completed using an audio computer-assisted interviewing system (Turner et al., 1998) on laptop computers (for adolescents) or on touch-screen tablet PCs (for parents). The system displays each item and response choices on the computer screen while the item and response choices are read to the participant through a set of headphones.

Following Knight, Roosa, and Umaña-Taylor (2009), rigorous tracking procedures were used to maintain contact with participants between assessment time points. At baseline, we obtained the names and contact information for three "contact persons" who would know how to reach the family if we were unable to do so. Names and phone numbers for these contact persons were updated at each assessment time point. Our assessors also called each family approximately once every 2 3 months to say hello and to ensure that our current contact information for the family was still correct. As a result of these tracking procedures, we were able to retain $85 \%$ of the sample ( 256 of the original 302 families) across the five waves included in the present analyses.

\section{Measures}

\section{Acculturation}

Consistent with Schwartz, et al. (2010), we assessed acculturation in terms of Hispanic and U.S. practices, values, and identifications.

Cultural practices were assessed using the Bicultural Involvement Questionnaire (BIQ; Szapocznik, Kurtines, \& Fernandez, 1980). The BIQ consists of 42 items, 21 assessing U.S. practices (e.g., speaking English, eating American food, associating with American friends) and 21 assessing Hispanic practices (e.g., speaking Spanish, eating Hispanic food, associating with Hispanic friends). Cronbach's alpha coefficients at baseline were .91 for U.S. practices and .89 for Hispanic practices. A 5-point Likert 
scale, ranging from 1 (strongly disagree) to 5 (strongly agree), was used.

We measured cultural values in terms of individualism-collectivism. Individualism and collectivism were assessed using a 16-item measure ( 8 items for individualism and 8 items for collectivism) developed by Triandis and Gelfand (1998). A 5-point Likert scale was used, ranging from 1 (strongly disagree) to 5 (strongly agree). Sample items include: "I'd rather depend on myself than on others" (individualism) and "Family members should stick together, no matter what sacrifices are required" (collectivism). Alpha coefficients at baseline were .73 for individualism and .79 for collectivism.

Ethnic and U.S. identifications were assessed using the Multigroup Ethnic Identity Measure (MEIM; Roberts et al., 1999) and the American Identity Measure (Schwartz, Park, et al., 2012). The American Identity Measure was adapted from the MEIM, with "the United States" inserted in place of "my ethnic group." Cronbach's alphas at baseline were .88 for U.S. identity and .91 for ethnic identity.

Mental health outcomes were assessed in terms of self-esteem, optimism, prosocial behavior, and depressive symptoms. Self-esteem was measured using the Rosenberg (1968) Self-Esteem Scale. This measure consists of 10 items, 5 of which are worded positively (e.g., "I feel that I have a number of good qualities") and 5 of which are worded negatively (e.g., "All in all, I am inclined to think I am a failure"). Negatively worded items are reversecoded and summed with the positively worded items to create a total score for the scale $(\alpha=.74)$. This measure has been used widely with Spanishspeaking samples (Schmitt \& Allik, 2005).

Optimism was measured using the Children's Hope Scale (Edwards, Ong, \& Lopez, 2007). This measure, designed specifically for use with Hispanics, consists of six items assessing the extent to which young people are optimistic about their future. Sample items include "I can think of many ways to get the things in life that are most important to me." Cronbach's alpha at baseline in the present study was .86 .

Prosocial behavior was measured using an adapted version the Prosocial Tendencies Measure (Carlo \& Randall, 2002). The version that we used consists of 19 items assessing the extent to which participants typically help others in a number of situations-including altruistic (where the identity of the helper will not be known to the recipient), highly emotional, dire need, and when helping others will make the helper "look good." We derived a total score $(\alpha=.86)$ by summing responses to all 19 items. A sample item is: "I like helping others because it makes me look good." The Prosocial Tendencies Measure has been used successfully with Hispanic adolescents (Carlo, Knight, McGinley, Zamboanga, \& Jarvis, 2010).

Depressive symptoms were assessed using the Center for Epidemiologic Studies Depression (CES-D) scale (Radloff, 1977), designed to assess depressive symptoms in the general adolescent and adult population. The CES-D consists of 20 items asking how often various depressive symptoms (e.g., lack of appetite, difficulty sleeping, lethargy) occurred during the week prior to assessment. Cronbach's alpha was .93 in the present sample at baseline. The CES$\mathrm{D}$ has been translated into Spanish and used frequently with Hispanic samples (e.g., Todorova, Falcón, Lincoln, \& Price, 2010).

Family functioning was measured in terms of both parent-adolescent and whole-family relational processes. We assessed parent-adolescent relationships in terms of parental involvement, positive parenting (rewards for appropriate behavior), and communication. In terms of whole-family functioning, we assessed cohesion and communication.

Parental involvement and positive parenting were assessed using the Parenting Practices Scale (Gorman-Smith, Tolan, Zelli, \& Huesmann, 1996). The parental involvement subscale consists of 12 items $(\alpha=.87)$, and the adolescent-reported positive parenting subscale consists of 6 items $(\alpha=.91)$. Sample items include: "How often did your parent ask you about your plans for the coming day?" (involvement) and "When you do something that your parent likes, does s/he give you a wink or a smile?" (positive parenting). The response scale for each item ranges from 1 (almost never) to 3 (often). Parent-adolescent communication was assessed using the 20-item Parent-Adolescent Communication Scale (baseline $\alpha=.93$; Barnes \& Olson, 1982). Sample items include: "I can express my feelings to my parent without feeling restrained." These measures have been used extensively with Hispanic adolescents (e.g., Schwartz et al., 2013).

Whole-family cohesion and communication were assessed using the Family Relations Scale (Tolan, Gorman-Smith, Huesmann, \& Zelli, 1997). The cohesion subscale consists of six items (baseline $\alpha=.79$ ) such as: "Family members feel very close to each other." The communication subscale consists of three items (baseline $\alpha=.67$ ) such as: "My family knows what I mean when I say something." The Family Relations Scale has been used extensively with Hispanic adolescents (e.g., Schwartz et al., 2013). 


\section{Results}

The present results are reported in three primary steps: Step 1, ascertaining the within-time associations among the various acculturation components; Step 2, determining the number of classes within each acculturation domain and combining the three sets of classes into one superordinate class variable; and Step 3, comparing these classes on the indices of mental health and family functioning described above.

\section{Step 1: Ascertaining Within-Time Associations Among Acculturation Components}

Table 2 reports within-time correlations between each pair of acculturation components. The various components were generally positively intercorrelated, and these correlations increased significantly between the first and last time points. These findings suggest that the structure of acculturation is becoming more closely defined as adolescents' time in the United States increases.

\section{Step 2: Determining the Number of Classes for Each Acculturation Domain}

Our first step in determining the number of classes within each acculturation domain was to determine whether growth in each domain could be considered to be linear, or whether a quadratic slope term would be needed to accurately represent the change patterns within a given domain. To accomplish this, we estimated models with both linear and quadratic slopes for both the U.S. and Hispanic components within each acculturation domain. Nonsignificant quadratic terms were dropped from the models, leaving only the intercept and linear slope for the components in question. The fit of the final model (after determining which quadratic terms to retain) was examined using four fit indices: the comparative fit index (CFI) and non-normed fit index (NNFI), which reflect the increase in fit for the specified model over a null model with no paths or latent variables, and the root mean square error of approximation (RMSEA) and the standardized root mean square residual (SRMR), which reflect the extent to which the covariance structure implied by the model deviates from the covariance structure observed in the data. The chi-square statistic is reported, but not used in interpretation, because it tests the null hypothesis of perfect fit to the data (which is rarely tenable in models with more than minimal degrees of freedom; West, Taylor, \& Wu, 2012). Kline (2010) suggests that CFI and NNFI values above .95, and RMSEA and SRMR values below .08, represent good model fit. The RMSEA also provides a $95 \%$ confidence interval.

Within each acculturation domain, after determining which quadratic terms to retain and testing the fit of the growth curve model, we ascertained the number of classes within that domain. As described by Nylund, Asparouhov, and Muthén (2007), multiple criteria should be used when deciding how many classes to extract in mixture models. First, the Vuong-Lo-Mendell-Rubin likelihood ratio test (LRT) indicates whether a solution with $k$ classes provides a significantly better fit compared to a solution with $k-1$ classes. Second, the entropy value $(E)$ indexes classification accuracy of the class solution as a whole, and should be $>.70$. Third, the posterior classification accuracy values for each individual class should also be $>.70$. Fourth, each class should represent at least $10 \%$ of the sample so that statistical comparisons between and among classes are adequately powered.

Because practices, values, and identifications represent conceptually distinct domains of acculturation, we estimated mixture models and conducted LRTs separately for each domain. Both Hispanic and U.S. acculturation components were included within each analysis. For each acculturation domain, we examined the extent to which the class solution was consistent across sites. We did this by comparing the fit of two models to the data: (a) an unconstrained model where the class solution was allowed to vary across sites and (b) a constrained model where the class solution was set equal across sites. A nonsignificant difference in fit between these two models indicates that the class solution is equivalent across sites. Because standard structural equation modeling fit indices are not available in models with latent classes, the two models can be compared using a formula involving the $-2 \log$ likelihood values for the two models. This formula provides a chi-square difference value where the difference in the numbers of parameters across the two models serves as the degrees of freedom (see Muthén \& Muthén, 2010, for further details).

\section{Cultural Practices}

Within the growth curve model for cultural practices, the quadratic slope for U.S. practices was statistically significant, $z=4.13, p<.001$, but the 
Table 2

Correlations Among Acculturation Components by Time Point

\begin{tabular}{|c|c|c|c|c|c|}
\hline Variable & Hispanic practices & Individualist values & Collectivist values & U.S. identity & Ethnic identity \\
\hline \multicolumn{6}{|l|}{ U.S. practices } \\
\hline Time 1 & $-.33^{* * *}$ & .09 & .09 & $.45^{* * *}$ & .04 \\
\hline Time 2 & $-.13^{*}$ & $.21^{* * *}$ & $.39^{* * *}$ & $.45^{* * *}$ & $.20^{* *}$ \\
\hline Time 3 & $.14^{*}$ & $.34^{* * *}$ & $.34^{* * *}$ & $.43^{* * *}$ & $.31^{* * *}$ \\
\hline Time 4 & $.15^{*}$ & $.30^{* * *}$ & $.39^{* * *}$ & $.47^{* * *}$ & $.24^{* * *}$ \\
\hline Time 5 & $.31^{* * *}$ & $.39^{* * *}$ & $.47^{* * *}$ & $.54^{* * *}$ & $.44^{* * *}$ \\
\hline Difference (T1 - T5) & $z=15.60^{* * *}, q=.67$ & $z=7.56^{* * *}, q=.32$ & $z=9.87^{* * *}, q=.42$ & $z=2.81^{* *}, q=.12$ & $z=10.16^{* * *}, q=.43$ \\
\hline \multicolumn{6}{|l|}{ Hispanic practices } \\
\hline Time 1 & - & .11 & $.31^{* * *}$ & -.06 & $.30^{* * *}$ \\
\hline Time 2 & & .09 & $.26^{* * *}$ & .04 & $.39^{* * *}$ \\
\hline Time 3 & & $.21^{* * *}$ & $.36^{* * *}$ & .08 & $.35^{* * *}$ \\
\hline Time 4 & & $.23^{* * *}$ & $.35^{* * *}$ & $.19^{* *}$ & $.36^{* * *}$ \\
\hline Time 5 & & $.22^{* * *}$ & $.44^{* * *}$ & $.19^{* *}$ & $.41^{* * *}$ \\
\hline Difference (T1 - T5) & & $z=2.66^{* *}, q=.11$ & $z=3.57^{* * *}, q=.15$ & $z=5.94^{* * *}, q=.25$ & $z=2.97^{* *}, q=.13$ \\
\hline \multicolumn{6}{|l|}{ Individualist values } \\
\hline Time 1 & & - & $.26^{* * *}$ & $.27^{* * *}$ & $.25^{* * *}$ \\
\hline Time 2 & & & $.30^{* * *}$ & $.39^{* * *}$ & $.24^{* * *}$ \\
\hline Time 3 & & & $.32^{* * *}$ & $.30^{* * *}$ & $.30^{* * *}$ \\
\hline Time 4 & & & $.39^{* * *}$ & $.40^{* * *}$ & $.29^{* * *}$ \\
\hline Time 5 & & & $.51^{* * *}$ & $.55^{* * *}$ & $.44^{* * *}$ \\
\hline Difference (T1 - T5) & & & $z=6.98^{* * *}, q=.30$ & $z=8.03^{* * *}, q=.34$ & $z=5.10^{* * *}, q=.22$ \\
\hline \multicolumn{6}{|l|}{ Collectivist values } \\
\hline Time 1 & & & - & $.24^{* * *}$ & $.47^{* * *}$ \\
\hline Time 2 & & & & $.46^{* * *}$ & $.47^{* * *}$ \\
\hline Time 3 & & & & $.33^{* * *}$ & $.54^{* * *}$ \\
\hline Time 4 & & & & $.38^{* * *}$ & $.49^{* * *}$ \\
\hline Time 5 & & & & $.50^{* * *}$ & $.64^{* * *}$ \\
\hline Difference (T1 - T5) & & & & $z=7.16^{* * *}, q=.30$ & $z=5.83^{* * *}, q=.25$ \\
\hline \multicolumn{6}{|l|}{ U.S. identity } \\
\hline Time 1 & & & & - & $.24^{* * *}$ \\
\hline Time 2 & & & & & $.35^{* * *}$ \\
\hline Time 3 & & & & & $.37^{* * * *}$ \\
\hline Time 4 & & & & & $.41^{* * *}$ \\
\hline Time 5 & & & & & $.55^{* * *}$ \\
\hline & & & & & $z=8.79^{* * *}, q=.37$ \\
\hline \multicolumn{6}{|l|}{ Ethnic identity } \\
\hline Time 1 & & & & & - \\
\hline Time 2 & & & & & \\
\hline Time 3 & & & & & \\
\hline Time 4 & & & & & \\
\hline Time 5 & & & & & \\
\hline
\end{tabular}

Note. The $q$ index of effect size represents the difference between the Fisher $z$ transformations for the two correlations in question. $* p<.05 . * * p .01 . * * * p .001$.

quadratic slope for Hispanic practices was not, $z=0.83, p=.41$. The quadratic slope for U.S. practices was negative, indicating that the growth rate decreased toward the end of the study. The final growth model for cultural practices fits the data well, $\chi^{2}(32)=73.71, p<.001, \mathrm{CFI}=.97, \mathrm{NNFI}=.96$, RMSEA $=.066,95 \%$ CI $[.046, .086]$, SRMR $=.058$.

The latent class growth analysis for cultural practices yielded a two-factor solution, LRT $=449.385$, $p<.05, E=.81$, posterior classification accuracy values ranged from .90 to .96 . In Class 1, both U.S. and Hispanic practices increased over time (with some leveling off in U.S. practices after Time 3), whereas in Class 2, both U.S. and Hispanic practices were largely stable over time. Accordingly, we named these classes increasing practices and stable practices respectively. Two hundred eight participants $(69 \%$ of the sample) were classified into the 
increasing practices classes, and 94 participants ( $31 \%$ of the sample) were classified into the stable practices class. Within the increasing practices class, Hispanic practices scores were higher than U.S. practices scores at all five time points ( $t$ values ranged from 6.95 to 11.41, all $p s<.001$ ), but the opposite was true in the stable practices class ( $t$ values ranged from 3.78 to 5.60 , all $p s<.001$ ). This class solution appeared to be consistent across sites, $\Delta \chi^{2}(10)=2.44, p=.99$. Figure 2 depicts this class solution over time.

\section{Cultural Values}

Within the growth curve model for cultural values, the quadratic slope for individualism was statistically significant, $z=2.20, p<.05$; however, the quadratic slope for collectivism was not, $z=1.41$, $p=.17$. The quadratic slope for individualism was positive, indicating that the growth rate increased toward the end of the study. The final growth model for cultural values fit the data well, $\chi^{2}(30)=45.53, \quad p<.04, \quad$ CFI $=.98, \quad$ NNFI $=.97$, RMSEA $=.041,95 \%$ CI [.012, .065], SRMR $=.059$.

The latent class growth analysis for cultural values yielded a two-factor solution, LRT $=454.173$, $p=.001, E=.83$, posterior classification accuracy values ranged from .93 to .96 . In Class 1, both individualist and collectivist values increased over time, whereas in Class 2, both individualist and collectivist values were largely stable over time. Accordingly, we named these classes increasing values and stable values, respectively. Seventy-one participants ( $24 \%$ of the sample) were classified into the increasing values classes, and 231 participants $(76 \%$ of the sample) were classified into the stable values class. At all time points, collectivism scores were significantly higher than individualism scores in both the increasing values class $(t$ values ranged from 4.40 to 6.81 , all $p s<.001)$ and the stable values class ( $t$ values ranged from 8.92 to 13.41, all ps $<.001)$. This class solution appeared to be consistent across sites, $\Delta \chi^{2}(3)=6.62, p=.09$. Figure 3 depicts this class solution over time.

\section{Cultural Identifications}

Within the growth curve model for cultural identifications, neither quadratic slope was statistically significant: U.S. identity, $z=0.69, p=.49$, or ethnic identity, $z=0.14, p=.89$. The final growth model for cultural identifications fits the data well, $\chi^{2}(37)=69.98, \quad p<.001, \quad$ CFI $=.96, \quad \mathrm{NNFI}=.95$, RMSEA $=.064,95 \%$ CI $[.034, .074]$, SRMR $=.065$.

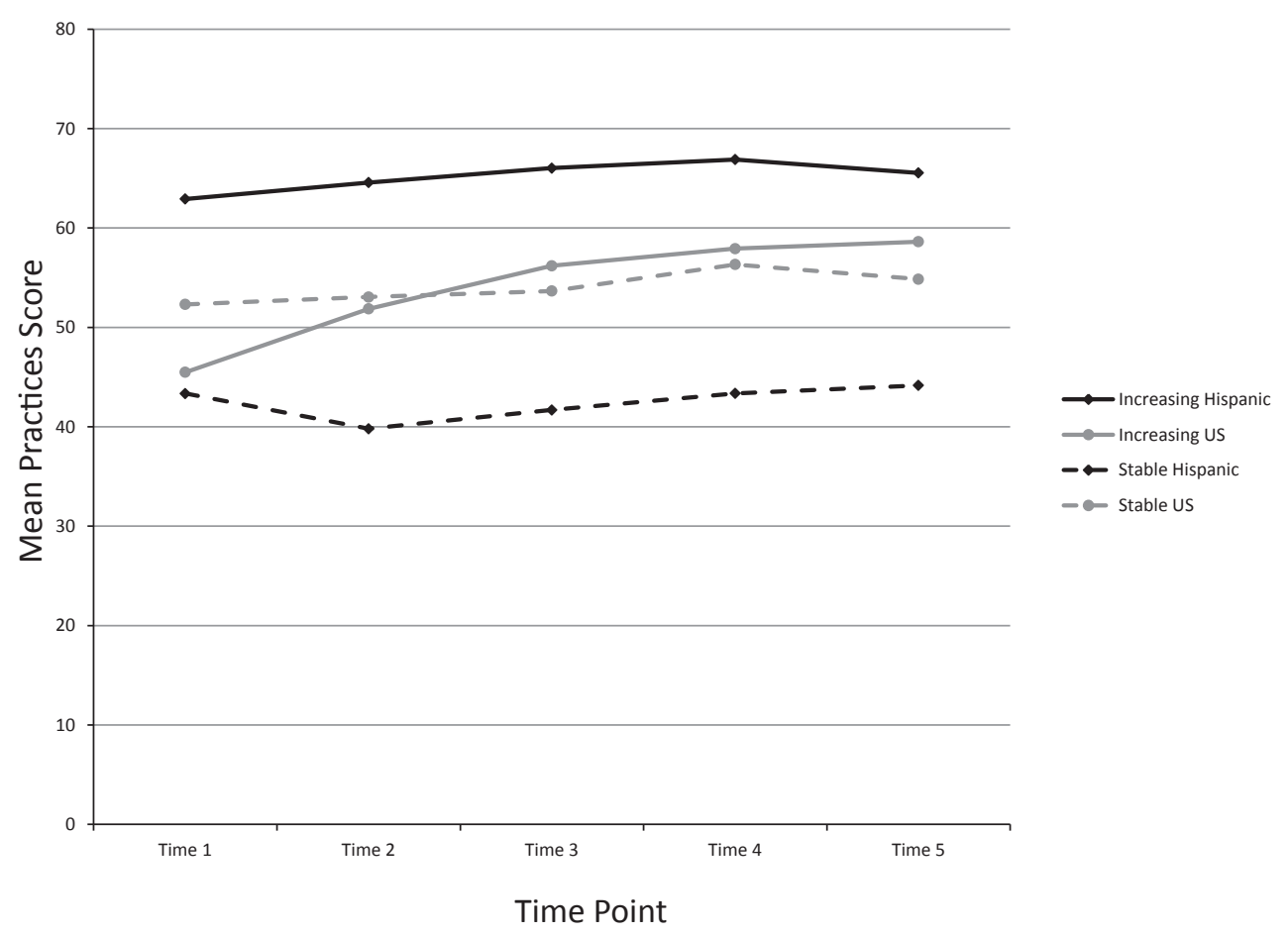

Figure 2. The two cultural practices classes clearly differ, with both U.S. and Hispanic practices higher in the increasing class than in the stable class for most of the study period. 


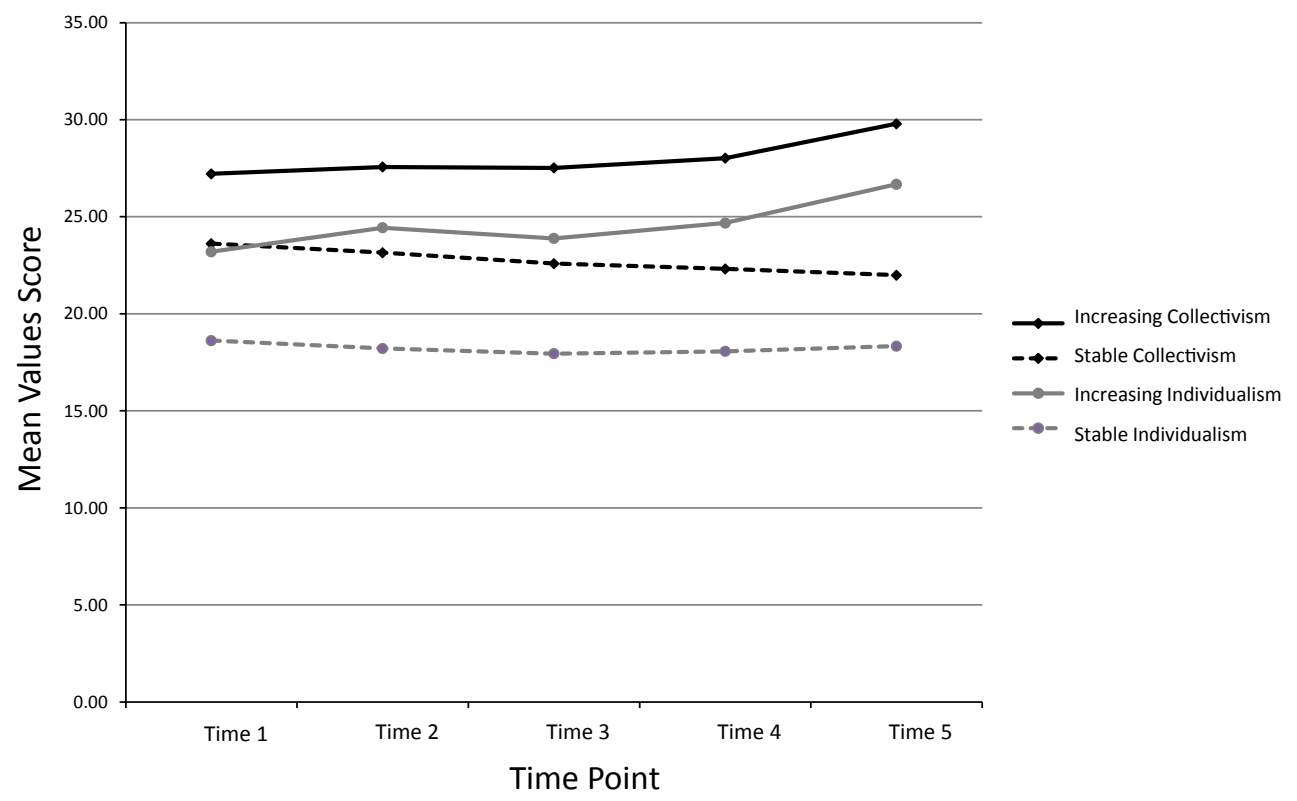

Figure 3. The cultural values solution clearly differentiates one class that increases in both individualism and collectivism from a second class that evidences little change in either of these value systems.

The latent class growth analysis for cultural identifications yielded a two-factor solution, LRT = $396.40, p=.01, E=.70$, posterior classification accuracy values ranged from .91 to .92. In Class 1, U.S. identity increased over time, and ethnic identity was high and stable over time, whereas in Class 2, both U.S. and ethnic identity were largely stable over time. Accordingly, we named these classes increasing identifications and stable identifications, respectively. One hundred forty-three participants ( $47 \%$ of the sample) were classified into the increasing identifications class, and 159 participants (53\% of the sample) were classified into the stable identifications class. In the increasing identifications class, ethnic identity scores were significantly higher than U.S. identity scores only at the first two time points ( $t$ values were 7.25 and 4.58 , both $p s<.001$ ). In the stable identifications class, ethnic identity scores were significantly higher than U.S. identity scores at the first four time points $(t$ values ranged from 3.91 to 5.63 , all $p \mathrm{~s}<.001$ ). Tests for differences in this class solution across sites approached significance, $\Delta \chi^{2}(3)=7.78, p=.051$. However, examinations of the class proportions at each site indicated that the proportion of participants assigned to each class differed by $<1 \%$ between the two sites. We therefore concluded that it was safe to assign participants to latent trajectory classes without stratifying by site. Figure 4 depicts this class solution over time.

\section{Creating Aggregated Classes Across Domains}

Our next step of analysis was to combine the class solutions for practices, values, and identifications to obtain a set of aggregated classes for acculturation over time. First, following Linda Muthén (Mplus discussion, April 9, 2013), and given that we were working with three sets of classes, within each class solution, we assigned each participant to her or his most likely class. Such a strategy was defensible given our high entropy values. Second, we cross-tabulated the two-class solutions for each of the three acculturation domains (see Table 3). The class with stable practices, increasing values, and stable identifications consisted of only two cases and was not used in analysis. We therefore had seven "combined" acculturation classes. Using $S$ for stable and I for increasing, and labeling the classes in terms of their standing on practices, values, and identifications (in that order), the classes are labeled as III, IIS, ISI, ISS, SII, SSI, and SSS. The III class $(n=49)$ increased over time in all three domains. The IIS class $(n=11)$ increased over time in practices and values but was stable over time in identifications. The ISI class $(n=65)$ increased over time in practices and identifications but was stable over time in values. The ISS class $(n=83)$ increased over time in practices but was stable over time in values and identifications. The SII 


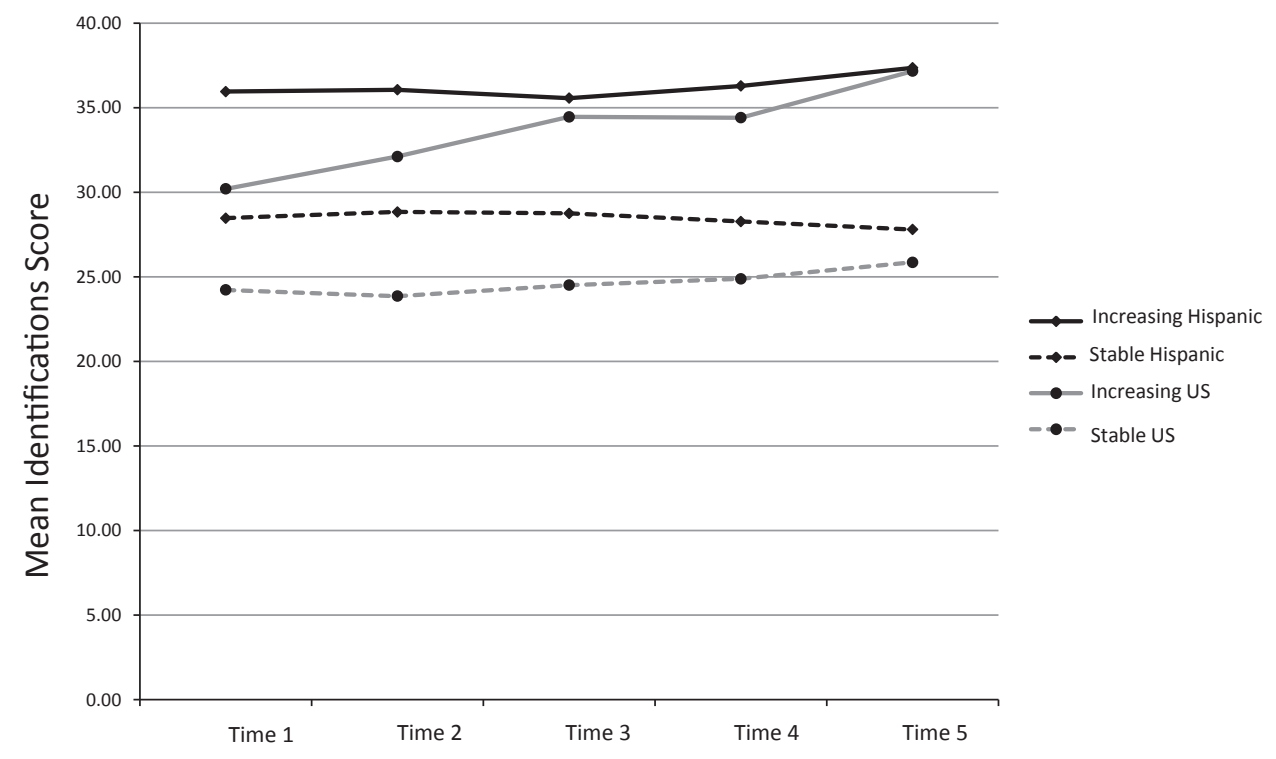

Time Point

Figure 4. The cultural identifications class solution consists of one class with higher and increasing U.S. and ethnic identity, and a second class with lower and stable levels of both U.S. and ethnic identity.

Table 3

Combined Acculturation Class Solution With Numbers of Participants in Each Class

\begin{tabular}{lccccc}
\hline & \multicolumn{2}{c}{ Increasing practices } & & \multicolumn{2}{c}{ Stable practices } \\
\cline { 2 - 3 } & Increasing values & Stable values & & Increasing values & Stable values \\
\hline Increasing identifications & 49 & 65 & 9 & 20 \\
Stable identifications & 11 & 83 & 2 & 63 \\
\hline
\end{tabular}

class $(n=9)$ increased over time in values but was stable over time in practices and identifications. The SSI class $(n=20)$ increased over time in identifications but was stable over time in practices and values. The SSS class $(n=63)$ was stable over time in all three domains. Figure 5 displays this class solution.

\section{Step 3: Patterns of Pairwise Differences on Outcome Variables}

Table 4 presents pairwise comparisons conducted across classes on each outcome variable. Baseline levels of each outcome variable were controlled in these analyses, such that the autocorrelation between baseline and Time 5 levels of the outcome variables was factored out of the class comparisons. This step is an important prerequisite for assuming directionality in the links between acculturation trajectories and adolescent mental health and family functioning (Cole \& Maxwell, 2003). For directional inferences to be safely drawn, an independent variable - in this case acculturation trajectories - must be predictive of the dependent variables even after accounting for earlier levels of these dependent variables.

In terms of additional statistical controls, the sandwich covariance estimator (Kauermann \& Carroll, 2001) was used to correct these comparisons for the nesting of adolescents within schools. We also controlled for gender, family income, years in the United States, and site in all of the comparisons across aggregated classes. Because of small sample sizes within some of the classes, we did not correct the significance levels for the number of tests conducted - such a strategy would have reduced statistical power even further (Kline, 2004). Such corrections are intended to reduce the likelihood of committing a Type I error-reporting a chance finding as statistically significant. However, it can be 


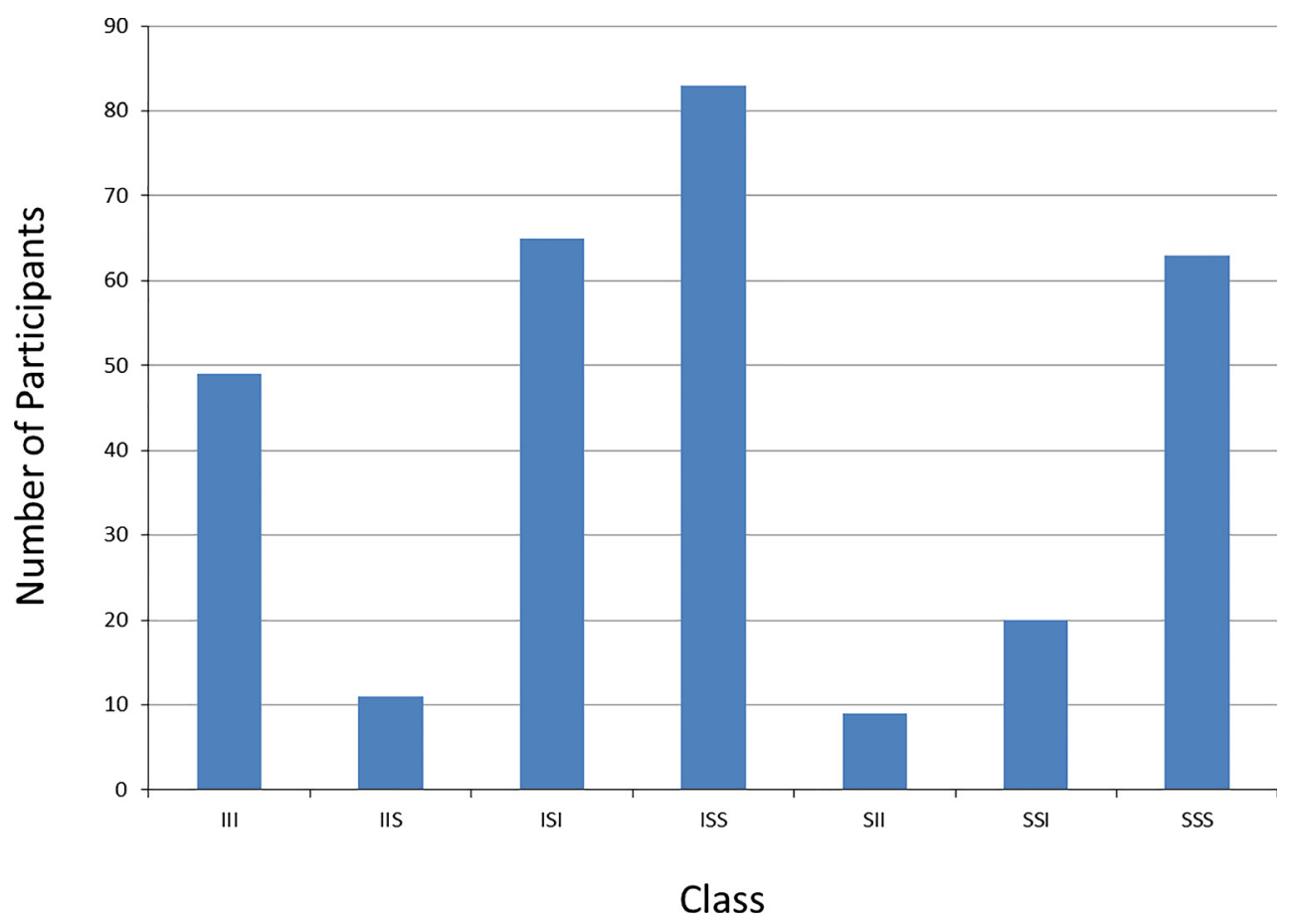

Figure 5. Combining the practices, values, and identifications solution yields seven superordinate classes. Using S for stable and I for increasing, and labeling the classes in terms of their standing on practices, values, and identifications (in that order), the classes are labeled as III, IIS, ISI, ISS, SII, SSI, and SSS.

Table 4

Means and Standard Deviations for Outcome Variables by Acculturation Trajectory Class

\begin{tabular}{|c|c|c|c|c|c|c|c|}
\hline \multirow[b]{2}{*}{ Outcome } & \multicolumn{7}{|c|}{ Acculturation trajectory class } \\
\hline & III & IIS & ISI & ISS & SII & SSI & SSS \\
\hline Self-esteem & $42.68_{a} \quad(6.45)$ & $42.25 \mathrm{a} \quad(4.98)$ & $40.57 \mathrm{a} \quad(6.12)$ & $40.25 \mathrm{a} \quad(7.04)$ & $45.00_{a} \quad(4.85)$ & $43.72_{\mathrm{a}} \quad(4.66)$ & $36.63_{\mathrm{b}} \quad(7.42)$ \\
\hline $\begin{array}{l}\text { Depressive } \\
\text { symptoms }\end{array}$ & $26.15_{\mathrm{ac}}(19.60)$ & $15.25 \mathrm{~b} \quad(14.28)$ & $28.96_{\mathrm{ac}}(15.25)$ & $30.31_{\mathrm{ac}}(13.66)$ & $22.10_{\mathrm{ab}}(19.37)$ & $19.94_{\mathrm{ab}}(11.52)$ & $35.12_{d}(12.37)$ \\
\hline Optimism & $26.60_{\mathrm{ac}}(5.00)$ & $26.50_{a} \quad(2.93)$ & $24.98_{\mathrm{ab}} \quad(4.49)$ & $22.31_{\mathrm{b}} \quad(5.67)$ & $28.00_{\mathrm{a}} \quad(2.21)$ & $26.61_{\mathrm{a}} \quad(2.33)$ & $19.65_{c} \quad(6.20)$ \\
\hline Prosocial behavior & $49.53_{a}(14.78)$ & $49.13 \mathrm{a} \quad(14.78)$ & $37.20_{\mathrm{b}} \quad(12.57)$ & $33.09_{\mathrm{b}} \quad(11.36)$ & $51.50 \mathrm{a} \quad(18.37)$ & $36.61_{\mathrm{b}} \quad(12.69)$ & $33.67_{b}(11.68)$ \\
\hline $\begin{array}{l}\text { Parent-adolescent } \\
\text { communication }\end{array}$ & 57.29 a $(14.05)$ & $53.17_{\mathrm{ab}}(11.44)$ & $52.05_{\mathrm{ab}}(16.48)$ & $48.38_{\mathrm{b}} \quad(12.97)$ & $46.83_{\mathrm{bc}}(18.80)$ & $55.03 \mathrm{a} \quad(11.17)$ & $43.71_{\mathrm{c}} \quad(8.86)$ \\
\hline Positive parenting & $28.48_{a} \quad(6.12)$ & $23.38_{\mathrm{bc}} \quad(9.13)$ & $22.80_{\mathrm{b}} \quad(6.94)$ & $21.46_{\mathrm{bd}}(7.16)$ & $27.20_{\mathrm{ac}}(6.09)$ & $21.83_{\mathrm{bd}} \quad(8.46)$ & $18.02_{\mathrm{d}} \quad(6.23)$ \\
\hline Parental involvement & $50.02_{\mathrm{a}} \quad(8.20)$ & $48.00_{\mathrm{ab}} \quad(9.22)$ & $42.22_{\mathrm{bc}}(11.98)$ & $40.03_{c} \quad(10.05)$ & $47.74_{\mathrm{ab}} \quad(9.17)$ & $46.81_{\mathrm{ab}} \quad(7.50)$ & $34.11_{\mathrm{d}}(10.22)$ \\
\hline $\begin{array}{l}\text { Overall family } \\
\text { cohesion }\end{array}$ & $16.13_{a} \quad(2.58)$ & $15.25 \mathrm{a} \quad(5.23)$ & $13.07_{\mathrm{b}} \quad(3.65)$ & $12.36_{\mathrm{b}} \quad(3.31)$ & $14.50_{\mathrm{ab}} \quad(4.40)$ & $12.06_{\mathrm{b}} \quad(3.73)$ & $10.20_{c} \quad(3.56)$ \\
\hline $\begin{array}{l}\text { Overall family } \\
\text { communication }\end{array}$ & $7.95 \mathrm{a} \quad(1.65)$ & $7.25_{\mathrm{ab}} \quad(3.11)$ & $6.57_{\mathrm{b}} \quad(2.02)$ & $6.10_{\mathrm{b}} \quad(1.83)$ & $7.10_{\mathrm{ab}} \quad(1.52)$ & $6.00_{\mathrm{b}} \quad(1.97)$ & $5.08_{c} \quad(2.10)$ \\
\hline
\end{tabular}

Note. Using S for stable and I for increasing, and labeling the classes in terms of their standing on practices, values, and identifications (in that order), the classes are labeled as III, IIS, ISI, ISS, SII, SSI, and SSS. Within each row, means that share a subscript do not differ significantly at $p<.05$. Pairwise comparisons were conducted adjusting for nesting of participants within schools and controlling for annual family income, gender, site, and years in the United States.

argued that the "best defense" against Type I error is a clear pattern of findings that are extremely unlikely to be due to chance. Highly similar patterns of class differences emerged for the nine outcome variables in our analyses, thereby producing such a clear pattern of findings. 
Patterns of pairwise differences are presented in Table 4. The classes with increasing levels of both practices and values (III and IIS) did not differ from one another on most of the outcome variables (all except depressive symptoms and positive parenting - the III class scored higher than the IIS class on both of these outcomes). The III class was among the highest on all of the indices of positive adjustment and family relationships, and the IIS class was lowest on depressive symptoms. The SII class, with stable practices and increasing values and identifications, generally scored high on indices of positive adjustment and family relationships, and low on depressive symptoms. The two classes with increasing practices and stable values (ISI and ISS) were also not significantly different on any of the outcome variables and scored second lowest (behind only the SSS class) on all of the positive adjustment and family relationship outcomes and second highest on depressive symptoms. The SSS class, with stable levels of practices, values, and identifications, scored lowest on all of the indices of positive adjustment and family relationships, and highest on depressive symptoms. No clear pattern emerged for the SSI class (stable practices and values, and increasing identifications).

To ensure that the class differences in mental health and family functioning variables at Time 5 were not redundant with class differences at baseline (i.e., that the class differences were truly developmental), we analyzed the pairwise differences at baseline and contrasted these patterns against the Time 5 patterns of pairwise differences. Results of these comparisons (available from the first author on request) indicated that the pairwise differences were greater at Time 5. In particular, the SSS class appeared to separate further from the other classes over time. For example, across outcome variables, the Cohen's $d$ effect size for the mean difference between the III and SSS classes was between $12 \%$ and $128 \%(M=77 \%)$ larger at Time 5 than at baseline.

\section{Discussion}

The present five-wave study was conducted to map the convergence among longitudinal trajectories of acculturation processes within three domainspractices, values, and identifications - within a sample of recently immigrated Hispanic adolescents, for whom acculturation might involve the most change over time. Within each acculturation domain, two classes emerged-one class characterized by increases in both Hispanic and U.S. cultural indices, and a second class characterized by relatively stable scores over time in both Hispanic and U.S. cultural indices. The finding that both Hispanic and U.S. cultural indices appeared to change in similar directions across all three acculturation domains (practices, values, and identifications) was somewhat consistent with Knight et al. (2009), who found that U.S. and Hispanic cultural practices tended to change similarly over time. Knight et al.'s (2013) study of changes in cultural values, however, found highly divergent patterns of change in U.S. versus Hispanic values over time. Our study was among the first to examine longitudinal trajectories of both ethnic and U.S. identity.

It should be noted that the samples used in most past studies have been composed primarily of longer term and second-generation immigrants, whereas the present sample was composed exclusively of recent immigrants. Within densely Hispanic areas of Miami and Los Angeles, young people are heavily exposed to both Hispanic and U.S. influences. Although many community residents communicate in Spanish, children and adolescents are educated in English, and media are widely available in both languages. Flannery, Reise, and $\mathrm{Yu}$ (2001) suggest that such highly bicultural contexts encourage children and adolescents to be bicultural.

The extent to which our results are consistent with a "bicultural context encouraging bicultural youth" hypothesis depends on the domain(s) in which biculturalism is operationalized. If the increasing classes are taken as indicative of "stronger" biculturalism, and if the stable classes are indicative of "weaker" or "more moderate" biculturalism, then the majority of adolescents in our sample were strongly bicultural in terms of practices, but only about half of the sample was strongly bicultural in terms of identifications, and less than a quarter of the sample was strongly bicultural in terms of values. The highly bicultural contexts in which our sample resided appear to have strongly encouraged biculturalism in terms of practices, but less so-at least within the $2 \frac{1}{2}$-year course of the present study-in terms of identifications and values. These results are consistent with Suárez-Orozco and Suárez-Orozco (2001) and Suárez-Orozco, Suárez-Orozco, and Todorova (2008), who found that immigrant adolescents tended to balance heritage and U.S. cultural practices, but to remain more loyal to the values and identifications of their cultural heritage. Among the three domains, practices may be most likely to 
change in the short term because immigrants need to interact with individuals from both their heritage-culture community (e.g., family members) and the larger U.S. society.

We should clarify how we are using the term biculturalism here. Although in most cases Hispanic acculturation components were endorsed more strongly than U.S. acculturation components were, this was not universally the case. In the increasing practices classes, the gap between endorsement of Hispanic and U.S. acculturation components became progressively smaller over time - and in the increasing identifications class, this gap was almost completely closed at the fifth time point. Certainly, within these two domains, individuals in the increasing classes appeared to manifest forms of biculturalism. Such a pattern was less evident within the values domain, where collectivism was endorsed significantly more strongly than individualism throughout the study period. The role of collectivism in Hispanic cultural streams may have contributed to this finding (see Smokowski \& Bacallao, 2011, for a more detailed review).

\section{Patterns of Overlap in Change Patterns Across Acculturation Domains}

We then cross-tabulated the three sets of trajectory classes with each to create a set of "superordinate" acculturation trajectory classes. The crosstabulations highlighted a number of patterns. First, whereas the majority of adolescents $(69 \%)$ reported that their endorsement of Hispanic and U.S. practices increased over time, the majority of adolescents $(76 \%)$ reported that their endorsement of individualist and collectivist values remained stable over time. The percentages of adolescents reporting increasing versus stable identifications over time were fairly equivalent ( $47 \%$ vs. 53\%). Following Schwartz, Vignoles, Brown, and Zagefka (2014), this pattern suggests that, following immigration, cultural practices may be the first component of acculturation that begins to change, and that values may be least likely to change during the first few years following immigration.

Indeed, most adolescents attend school in the society of settlement and must learn the language of that society. Other cultural practices, such as food and friendship preferences, may also change as immigrant adolescents begin to interact with their new context. Interestingly, Hispanic cultural practices also increase somewhat during the years following immigration for many adolescents. Adolescents who live in ethnic enclaves may take pride in their heritage and in opportunities to continue engaging in practices reflecting that heritage. Most likely, newer immigrants reinforce the heritage of others who have been in the United States longer, keeping the heritage cultural stream alive within the community. It is also possible that heritage-cultural retention helps adolescents to cope with the stressors of arriving in a new country with a new set of cultural expectations.

Still another possibility is that, as they spend more time in the United States, Hispanic immigrants may come to recognize their original behaviors as "Hispanic." In cross-cultural psychology, such a phenomenon is referred to as the "frame of reference effect" (Smith, Fischer, Vignoles, \& Bond, 2013). Cultural practices are often taken for granted when one is operating within a context where those behaviors are normative, but those behaviors are viewed as "cultural" when one is operating within a new context where one's typical behaviors are different from the norm. Berry (1997) hypothesized that acculturation results from contact with others who are culturally dissimilar from oneself, and he suggested that such contact would cause the person to examine her or his own cultural attitudes as well as those held by others. At least in adolescence, and within bicultural contexts that encourage endorsement of both one's cultural heritage and of the receiving culture, acculturation may be likely to manifest as similar patterns of change in endorsement of both cultural streams.

Values, on the other hand, may take more time to change - if they change at all. One's core beliefs about the prioritization of the individual vis-à-vis the family or other social group may be among the strongest components of cultural identity (Triandis, 1995). Many Hispanic cultural streams, for example, are deeply rooted in collectivist-based value systems such as familismo (priority placed on obligations to family members) and respeto (respect for others). These value systems may be unlikely to change after only a few years in the United Statesand to the extent to which the immigrant resides within a coethnic community, changes in core values may be met with some degree of disapproval. Individualism and collectivism are not inherently incompatible (Oyserman, Coon, \& Kemmelmeier, 2002); for example, one can be individualist at school or work and collectivist at home-adolescents may not perceive a need to change their cultural values.

Identifications appeared to be moderately likely to change across time. Immigrant adolescents may begin to identify with the United States, but as they 
do so, they increase their identification with their heritage cultures as well. Some of this biculturalism may be due to the communities in which these adolescents and their families have settled: All of the schools from which we recruited were at least $75 \%$ Hispanic, suggesting that the neighborhood in which these adolescents lived were also heavily Hispanic. Kiang and Fuligni (2009) have found that associating with coethnic friends, and with family members who have lived in the United States for longer periods of time, may help to maintain or increase one's endorsement of ethnic identity over time. It is also possible that ethnic identity increases following immigration as adolescents begin to emphasize themselves as Cuban, Mexican, Colombian, and so on, within the United States. For example, individuals living in Colombia likely do not spend a great deal of time thinking of themselves as Colombian, but the frame of reference effect suggests that identification as Colombian might increase following immigration to the United States.

It is important to note that none of the classes we found involved decreases in U.S. or Hispanic practices, values, or identifications. Acculturation components either increased or remained stable throughout the study period. Such a pattern of findings may suggest that exposure to a new cultural context tends to be an additive, rather than subtractive, experience. That is, adolescents can incorporate a second cultural stream into their cultural repertoire. Such a finding is consistent with Cheung et al. (2011), who found that childhood and adolescence represent a "sensitive period" for cultural adaptation. Our findings also support Nguyen and Benet-Martínez (2013), who identified biculturalism as the most advantageous approach to acculturation across a variety of life outcomes. Adolescence might be the ideal time to begin incorporating a second cultural stream, given that (a) the person has spent enough time in the culture of origin to retain it and (b) that the person is still young enough to fluidly incorporate a second cultural stream.

Biculturalism may also represent an expression of identity development for Hispanic adolescents, and perhaps for those from other minority and immigrant groups as well (Cooper, 2011; Fuller \& García Coll, 2010; Marks, Szalacha, Lamarre, Boyd, \& García Coll, 2007). Biculturalism allows Hispanic adolescents to "live in two worlds" - the traditional world of their parents and the mainstream world of Americanized peers and media (Schwartz \& Zamboanga, 2008). Immigrant and minority adolescents are confronted with the task of developing a sense of identity that balances their cultural heritage with U.S. culture. Developing such a bicultural identity might therefore be considered as a key developmental task for adolescents from immigrant and minority groups, and there are at least three domains in which a bicultural identity can be developed. The present results suggest that, at least in the short term, some adolescents are more successful than others in terms of addressing this developmental task - and that their mental health and family relationships may vary as a function of this success.

\section{Acculturation Trajectory Classes and Outcome Variables}

We then combined the three sets of trajectory classes into one set of "superordinate" trajectory classes, where each superordinate class was defined as a combination of change trajectories for practices, for values, and for identifications (see Figure 4). We examined a number of mental health and family functioning indices across these classes. Within the domain of mental health, we included self-esteem, depressive symptoms, optimism, and prosocial behavior. Within the domain of family functioning, we included parent-adolescent involvement, positive parenting, and communication, as well as overall family cohesion and communication. These "outcome" variables were included at the fifth study time point, with baseline values controlled (see Table 5).

As a general rule, the class that was stable over time on all three acculturation domains (the SSS class) reported the least favorable levels of all of the mental health and family functioning variables. Also as a general rule, one or both of the classes characterized by increasing practices and values (the III and/or IIS classes) scored among the most favorable on all of the outcome variables. The SII class (stable practices, increasing values and identifications) also tended to score favorably. It may be that failure to acculturate in any way (the SSS class) serves as an index of maladaptation, and that increases in at least two of the acculturation domains are important in facilitating adaptive mental health and family functioning. If acculturation among recent-immigrant adolescents is represented as increases over time in one or more cultural domains, then lack of change in any of these domains (i.e., the SSS class) may be analogous to Berry's (1997) marginalization category (where the person rejects both the heritage and receiving cultures). Marginalization is often associated with the poorest psychosocial outcomes 
Table 5

Summary of Findings

Outcome variable

Self-esteem

Depressive symptoms

Optimism

Prosocial behavior

Parent-adolescent communication

Positive parenting

Parental involvement

Overall family cohesion

Overall family communication
Patterns of differences

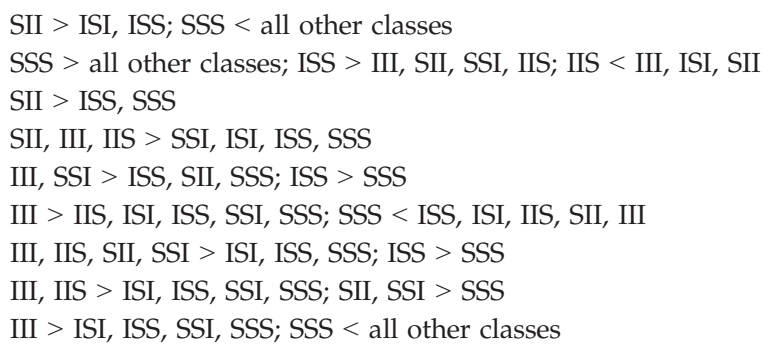

Note. Using $\mathrm{S}$ for stable and I for increasing, and labeling the classes in terms of their standing on practices, values, and identifications (in that order), the classes are labeled as III, IIS, ISI, ISS, SII, SSI, and SSS.

(Berry, Phinney, Sam, \& Vedder, 2006). As a case in point, mean differences in all of the mental health and family functioning variables between the SSS class and the other classes increased over the course of the study-suggesting that this form of marginalization is not only linked with the least favorable outcomes, but that this link with unfavorable outcomes is exacerbated as adolescents spend more time in the United States.

One or both of the classes characterized by increasing practices and stable values (the ISI and ISS classes) tended to indicate less favorable functioning at the final time point compared to the classes characterized by increases in both practices and values. This general trend held for all of the mental health and family functioning variables, although the specific pattern of pairwise differences (i.e., III or IIS vs. ISI or ISS) varied across the outcome variables. What this set of findings appears to suggest is that increases in at least two acculturation domains - especially practices and values - is facilitative of more favorable family functioning and mental health outcomes.

The role of identifications appeared to be inconsistent across outcome variables, except for the difference between the SSI and SSS classes (this difference was significant for all of the outcome variables except for prosocial behavior and positive parenting). This pattern of results suggests that whether ethnic and U.S. identity increase or remain stable does not have a consistent effect on mental health or family functioning unless practices and values are both stable. Only when practices and values are both stable do increasing identifications predict higher self-esteem, fewer depressive symptoms, higher optimism, more effective parentadolescent communication, stronger parental involvement, and higher levels of overall family cohesion and communication. We might therefore conclude that changes in identifications are somewhat less important than changes in practices and values, in terms of their effects on reports of mental health and family functioning among recently immigrated Hispanic adolescents. This pattern may indicate that fluctuations in cultural identifications -which may be expected in adolescence as young people explore different possibilities-are normative (at least in moderation) and may not compromise mental health or family functioning.

Theories of ethnic identity may also help to explain the somewhat modest role of identifications in the present results. Ethnic identity (and by extension, U.S. identity) consists of both an affective component and a behavioral component (Phinney \& Ong, 2007; Umaña-Taylor et al., 2014). That is, identifying with one's heritage cultural group, the United States, or both involves thinking about the meaning of the group membership, as well as participating in activities reflective of the group. The behavioral component of ethnic identity may overlap with cultural practices, such that only the affective component contributes uniquely to predicting outcomes.

\section{Strengths and Limitations of the Present Study}

The present study is characterized by a number of strengths and limitations that should be considered when interpreting the present results. At least five strengths should be highlighted. First, the fivewave longitudinal design allowed us to estimate fairly precise growth trajectories. Second, the inclusion of both Miami and Los Angeles provided a more diverse and representative sample than would 
have been possible at either site alone. Third, the sample of recent immigrants provided us with a sample that was likely engaged in a rapid period of change in acculturative processes than would have been the case with a sample of long-term or second-generation immigrants. Fourth, the use of a bidimensional, multidomain model of acculturation permitted us to map the intersections among three domains and to create a more complex (and likely more accurate) picture than would have emerged had we considered only a single domain. Indeed, much of the acculturation literature has relied on single-domain measures of acculturation (especially cultural practices). Our results suggest that change patterns in cultural practices, values, and identifications are different enough from each other that including only one of these domains is unlikely to capture the full impact of acculturation trajectories on mental health and family functioning in recently immigrated Hispanic adolescents.

In terms of limitations, the use of data from a single reporter may have inflated the relations between acculturative processes and outcome variables. However, the use of a longitudinal design where the acculturation and outcome variables were modeled together at only two of the five time points may temper this limitation to some extent. Second, it may be useful to triangulate self-reports of family processes with independent observer ratings of family interactions (Sessa, Avenevoli, Steinberg, \& Morris, 2001). Third, we were more successful recruiting and retaining participants in Miami, where Cubans become legal residents upon arrival in the United States, than in Los Angeles, where many Mexican immigrants are undocumented. We do not know how our results might have changed had we been able to capture the experiences of those undocumented families who did not participate in our study due to fear of deportation. Fourth, although quantitative surveys can provide a great deal of information about the acculturation process, a qualitative component would have offered additional insights into adolescents' first-person experiences. Especially with regard to acculturation, which represents the intersection of multiple cultural streams, qualitative methods work can provide a culturally grounded, emic perspective that is less accessible to quantitative-based methods (Falicov, 2013). Fifth, the use of two highly bicultural contexts did not allow us to examine how immigrant adolescents might have acculturated, and how this acculturation would have mapped onto mental health and family outcomes, in more monocultural settings. Further, empirically measuring perceived context of reception would allow for examination of ways in which the atmosphere into which one is migrating would affect one's patterns of acculturation (Schwartz, Unger, et al., 2014).

\section{Conclusions and Implications for Intervention}

In light of the study strengths, and in spite of the study limitations, our results may have important implications for further research, as well as for intervention development and refinement. Researchers (e.g., Smokowski \& Bacallao, 2011; Szapocznik et al., 1986) have identified promoting biculturalism as an important goal for culturally based interventions, and the present results echo such recommendations. However, much work remains to be done in terms of developing and refining such interventions. In highly bicultural and multicultural settings such as Miami and Los Angeles, endorsement of multiple cultural streams may be the most favorable approach to acculturation (Schwartz \& Unger, 2010). The present findings support such a contention, given that, within each cultural domain, increases in the U.S. component tended to be accompanied by increases in the matching Hispanic component. The convergence between Hispanic and U.S. components within the increasing practices and identifications classes suggests that a form of biculturalism may be developing in some recently immigrated adolescents. It appears, however, that increases in Hispanic and U.S. cultural practices, without corresponding increases in individualist and collectivist values, may present a risk for somewhat compromised mental health and family relationships. That is, changes in practices, without corresponding changes in values, may not be adaptive - at least within the bicultural and multicultural contexts in which our study was conducted.

Interventions to promote biculturalism in immigrants, then, might focus not only on promoting comfort with heritage and receiving cultural practices and values (and identifications to a somewhat lesser extent) - but also on becoming comfortable integrating one's heritage and receiving cultural streams. Especially in bicultural and multicultural areas - which are becoming more and more common in the United States and other Western countries-a bicultural orientation may be important vis-à-vis family relationships and mental health outcomes among adolescent and emerging adult immigrants. Moreover, the present results (as well as the multidimensional model of acculturation on which our study is based) suggest that biculturalism may take 
different forms across the three acculturation domains. Promoting biculturalism in the practices domain, for example, may require different intervention strategies than would promoting biculturalism in the values or identifications domains. It is our hope that the present study will inspire more work in developing and evaluating interventions to help adolescent immigrants to become bicultural, and in doing so, helping to promote positive mental health and family relationships in adolescent immigrants. The fields of prevention science and positive youth development may provide some guidance in designing such interventions and policies (e.g., Ferrer-Wreder, 2014; Fuller \& García Coll, 2010). It is essential that our interventions and policies catch up with the rapidly increasing diversity of our populations.

\section{References}

Barnes, H. L., \& Olson, D. H. (1982). Parent-adolescent communication and the circumplex model. Child Development, 56, 438-447. doi:10.2307/1129732

Bernstein, R. (2013). U.S. Census Bureau projections show a slower growing, older, more diverse nation a half century from now. Retrieved from https://www.census.gov/news room/releases/archives/population/cb12-243.html

Berry, J. W. (1997). Immigration, acculturation, and adaptation. Applied Psychology: An International Review, 46, 5-34. doi:10.1080/026999497378467

Berry, J. W., Phinney, J. S., Sam, D. L., \& Vedder, P. (2006). Immigrant youth in cultural transition. Mahwah, NJ: Erlbaum.

Carlo, G., Knight, G. P., McGinley, M., Zamboanga, B. L., \& Jarvis, L. H. (2010). The multidimensionality of prosocial behaviors and evidence of measurement equivalence in Mexican American and European American early adolescents. Journal of Research on Adolescence, 20, 334-358. doi:10.1111/j.1532-7795.2010.00637.x

Carlo, G., \& Randall, B. A. (2002). The development of a measure of prosocial behaviors for late adolescents. Journal of Youth and Adolescence, 31, 31-44. doi:10.1023/ A:1014033032440

Castillo, L. G., \& Caver, K. (2009). Expanding the concept of acculturation in Mexican American rehabilitation psychology research and practice. Rehabilitation Psychology, 54, 351-362. doi:10.1037/a0017801

Cheung, B. Y., Chudek, M., \& Heine, S. J. (2011). Evidence for a sensitive period for acculturation: Younger immigrants report acculturating at a faster rate. Psychological Science, 22, 147-152. doi:10.1177/0956797 610394661

Cole, D. A., \& Maxwell, S. E. (2003). Testing mediational models with longitudinal data: Questions and tips in the use of structural equation modeling. Journal of
Abnormal Psychology, 112, 558-577. doi:10.1037/0021843X.112.4.558

Cooper, C. R. (2011). Bridging multiple worlds: Cultures, identities, and pathways to change. Oxford, UK: Oxford University Press.

Edwards, L. M., Ong, A. D., \& Lopez, S. J. (2007). Hope measurement in Mexican American youth. Hispanic Journal of Behavioral Sciences, 29, 225-241. doi:10.1177/ 0739986307299692

Ennis, S. R., Rios-Vargas, M., \& Albert, N. G. (2011). The Hispanic population, 2010 (Census brief C2010BR-4). Washington, DC: U.S. Census Bureau.

Falicov, C. J. (2013). Latino families in therapy (2nd ed.). New York, NY: Guilford Press.

Ferrer-Wreder, L. (2014). Advancing child and adolescent well-being through positive youth development and prevention programs. In A. Ben-Asch, F. Casas, I. Frønes, \& J. E. Korbin (Eds.), Handbook of child wellbeing: Theories, methods, and policies in global perspective (pp. 3025-3041). New York, NY: Springer.

Flannery, W. P., Reise, S. P., \& Yu, J. (2001). A comparison of acculturation models. Personality and Social Psychology Bulletin, 27, 1035-1045. doi:10.1177/014616 7201278010

Forster, M., Grigsby, T. J., Soto, D. W., Schwartz, S. J., \& Unger, J. B. (2014). The role of bicultural stress and perceived context of reception in the expression of aggression and rule breaking behaviors among newimmigrant Hispanic youth. Journal of Interpersonal Violence. Advance online publication. doi:10.1177/08862 60514549052

Fuller, B., \& García Coll, C. (2010). Learning from Latinos: Contexts, families, and child development in motion. Developmental Psychology, 46, 559-565. doi:10. 1037/a0019412

García Coll, C., Akerman, A., \& Cicchetti, D. (2000). Cultural influences on developmental processes and outcomes: Implications for the study of development and psychopathology. Development and Psychopathology, 12, 333-356. doi:10.1017/S0954579400003059

Gonzales, N. A., Deardorff, J. M., Formoso, D., Barr, A., \& Barrera, M., Jr. (2006). Family mediators of the relation between acculturation and adolescent mental health. Family Relations, 55, 318-330. doi:10.1111/j.17413729.2006.00405.x

González-Wahl, A. M., \& McNulty-Eitle, T. (2010). Gender, acculturation, and alcohol use among Latina/o adolescents: A multi-ethnic comparison. Journal of Immigrant and Minority Health, 12, 153-165. doi:10.1007/ s10903-008-9179-6

Gordon, M. (1964). Assimilation in American life. New York, NY: Oxford University Press.

Gorman-Smith, D., Tolan, P. H., Zelli, A., \& Huesmann, L. R. (1996). The relation of family functioning to violence among inner-city minority youths. Journal of Family Psychology, 10, 115-129. doi:10.1037/0893-3200.10.2.115

Grieco, E. M., Trevelyan, E., Larsen, L., Acosta, Y. D., Gambino, C., de la Cruz, G. P., . . . Walters, N. (2012). 
The size, place of birth, and geographic distribution of the foreign-born population in the United States: 1960 to 2010 (Population Division Working Paper 96). Washington, DC: U.S. Census Bureau.

Hayes-Bautista, D. (2004). La Nueva California: Latinos in the Golden State. Berkeley: University of California Press.

Henderson, T. J. (2011). Beyond borders: A history of Mexican migration to the United States. Malden, MA: WileyBlackwell.

Hirschman, E. C. (2003). Men, dogs, guns, and cars: The semiotics of rugged individualism. Journal of Advertising, 32, 9-22.

Huntington, S. P. (2004). Who are we? Challenges to America's national identity. New York, NY: Simon \& Schuster.

Kasinitz, P. A., Mollenkopf, J., Waters, M., \& Holdaway, J. (2008). Inheriting the city: The children of immigrants come of age. Thousand Oaks, CA: Sage.

Kauermann, G., \& Carroll, R. J. (2001). A note on the efficiency of sandwich covariance matrix estimation. Journal of the American Statistical Association, 96, 1387-1398.

Kiang, L., \& Fuligni, A. J. (2009). Ethnic identity in context: Variations in ethnic exploration and belonging within parent, same-ethnic peer, and different-ethnic peer relationships. Journal of Youth and Adolescence, 38, 732-743. doi:10.1007/s10964-008-9278-7

Kline, R. B. (2004). Beyond significance testing: Reforming data analysis in behavioral research. Washington, DC: American Psychological Association.

Kline, R. B. (2010). Principles and practices of structural equation modeling (3rd ed.). New York, NY: Guilford Press.

Knight, G. P., Basilio, C. D., Cham, H., Gonzales, N. A., Liu, Y., \& Umaña-Taylor, A. J. (2013). Trajectories of Mexican American and mainstream cultural values among Mexican American adolescents. Journal of Youth and Adolescence. Advance online publication. doi:10. 1007/s10964-013-9983-8

Knight, G. P., Gonzales, N. A., Saenz, D. S., Bonds, D. D., Germán, M., Deardorff, J., . . . Updegraff, K. A. (2010). The Mexican American Cultural Values Scale for adolescents and adults. Journal of Early Adolescence, 30, 444-481. doi:10.1177/0272431609338178

Knight, G. P., Roosa, M. W., \& Umaña-Taylor, A. J. (2009). Methodological challenges in studying ethnic minority or economically disadvantaged populations. Washington, DC: American Psychological Association.

Knight, G. P., Vargas-Chanes, D., Losoya, S. H., Cota-Robles, S., Chassin, L., \& Lee, J. (2009). Acculturation and enculturation trajectories among Mexican-American adolescent offenders. Journal of Research on Adolescence, 19, 625-653. doi:10.1111/j.1532-7795.2009.00614.x

LaFromboise, T., Coleman, H. L. K., \& Gerton, J. (1993). Psychological impact of biculturalism: Evidence and theory. Psychological Bulletin, 114, 395-412. doi:10.1037/ 0033-2909.114.3.395

Lau, A. S., McCabe, K. M., Yeh, M., Garland, A. F., Wood, P. A., \& Hough, R. L. (2005). The acculturation gap-distress hypothesis among high-risk Mexican
American families. Journal of Family Psychology, 19, 367375. doi:10.1037/0893-3200.19.3.367

Lerner, R. M., \& Galambos, N. L. (1998). Adolescent development: Challenges and opportunities for research, programs, and policies. Annual Review of Psychology, 49, 413-446. doi:10.1146/annurev.psych.49.1. 413

Light, I. H. (2006). Deflecting immigration: Networks, markets, and regulation in Los Angeles. New York, NY: Russell Sage Foundation.

Marks, A. K., Szalacha, L. A., Lamarre, M., Boyd, M. J., \& García Coll, C. (2007). Emerging ethnic identity and interethnic group social preferences in middle childhood: Findings from the Children of Immigrants Development in Context (CIDC) study. International Journal of Behavioral Development, 31, 501-513. doi:10.1177/ 0165025407081462

Muthén, L. K., \& Muthén, B. O. (2010). Mplus user's guide, version 6. Los Angeles, CA: Author.

Nguyen, A. M. D., \& Benet-Martínez, V. (2013). Biculturalism and adjustment: A meta-analysis. Journal of CrossCultural Psychology, 44, 122-159. doi:10.1177/ 0022022111435097

Nylund, K., Asparouhov, T., \& Muthén, B. (2007). Deciding on the number of classes in latent class analysis and growth mixture modeling: A Monte Carlo simulation study. Structural Equation Modeling, 14, 535-569. doi:10.1080/10705510701575396

Oyserman, D., Coon, H. M., \& Kemmelmeier, M. (2002). Rethinking individualism and collectivism: Evaluation of theoretical assumptions and meta-analyses. Psychological Bulletin, 128, 3-72. doi:10.1037/0033-2909.128.1.3

Pew Hispanic Center. (2010). Public supports Arizona immigration law. Retrieved from http://pewresearch.org/ pubs/1591/public-support-arizona-immigration-lawpoll

Phinney, J. S., \& Ong, A. D. (2007). Conceptualization and measurement of ethnic identity: Current status and future directions. Journal of Counseling Psychology, 54, 271-281. doi:10.1037/0022-0167.54.3.271

Portes, A., \& Rumbaut, R. G. (2006). Immigrant America: A portrait (3rd ed.). Berkeley: University of California Press.

Radloff, L. (1977). The CES-D scale: A self-report depression scale for research in the general population. Applied Psychological Measurement, 1, 385-401.

Roberts, R. E., Phinney, J. S., Masse, L. C., Chen, Y. R., Roberts, C. R., \& Romero, A. (1999). The structure of ethnic identity in young adolescents from diverse ethnocultural groups. Journal of Early Adolescence, 19, 301322. doi:10.1177/0272431699019003001

Rosenberg, M. (1968). Society and the adolescent self-image. Princeton, NJ: Princeton University Press.

Rumbaut, R. G. (2008). Reaping what you sow: Immigration, youth, and reactive ethnicity. Applied Developmental Science, 12, 108-111. doi:10.1080/10888690801997341

Sam, D. L., \& Berry, J. W. (2010). Acculturation: When individuals and groups of different cultural back- 
grounds meet. Perspectives on Psychological Science, 5, 472-481. doi:10.1177/1745691610373075

Schmitt, D. P., \& Allik, J. (2005). Simultaneous administration of the Rosenberg Self-Esteem Scale in 53 nations: Exploring the universal and culture-specific features of global self-esteem. Journal of Personality and Social Psychology, 89, 623-642. doi:10.1037/0022-3514.89. 4.623

Schwartz, S. J., Benet-Martínez, V., Knight, G. P., Unger, J. B., Zamboanga, B. L., Des Rosiers, S. E., . . . Szapocznik, J. (2014). Effects of language of assessment on the measurement of acculturation: Measurement equivalence and cultural frame switching. Psychological Assessment, 26, 100-114. doi:10.1037/a0034717

Schwartz, S. J., Des Rosiers, S. E., Huang, S., Zamboanga, B. L., Unger, J. B., Knight, G. P., . . . Szapocznik, J. (2013). Developmental trajectories of acculturation in Hispanic adolescents: Associations with family functioning and adolescent risk behavior. Child Development, 84, 1355-1372. doi:10.1111/cdev.12047

Schwartz, S. J., Pantin, H., Sullivan, S., Prado, G., \& Szapocznik, J. (2006). Nativity and years in the receiving culture as markers of acculturation in ethnic enclaves. Journal of Cross-Cultural Psychology, 37, 345353. doi:10.1177/0022022106286928

Schwartz, S. J., Park, I. J. K., Huynh, Q.-L., Zamboanga, B. L., Umaña-Taylor, A. J., Lee, R. M., . . Agocha, V. B. (2012). The American Identity Measure: Development and validation across ethnic subgroup and immigrant generation. Identity: An International Journal of Theory and Research, 12, 93-128.

Schwartz, S. J., \& Unger, J. B. (2010). Biculturalism and context: What is biculturalism, and when is it adaptive? Human Development, 53, 26-32. doi:10.1159/000268137

Schwartz, S. J., Unger, J. B., Des Rosiers, S. E., Huang, S., Baezconde-Garbanati, L., Lorenzo-Blanco, E., ... Szapocznik, J. (2012). Substance use and sexual behavior among recent Hispanic immigrant adolescents: Effects of parent-adolescent differential acculturation and communication. Drug and Alcohol Dependence, 125S, S26-S34. doi:10.1016/j.drugalcdep.2012.05.020

Schwartz, S. J., Unger, J. B., Lorenzo-Blanco, E., Des Rosiers, S. E., Villamar, J. A., Soto, D. W., . . . Szapocznik, J. (2014). Perceived context of reception among recent Hispanic immigrants: Conceptualization, instrument development, and preliminary validation. Cultural Diversity and Ethnic Minority Psychology, 20, 1-15. doi:10.1037/a0033391

Schwartz, S. J., Unger, J. B., Zamboanga, B. L., \& Szapocznik, J. (2010). Rethinking the concept of acculturation: Implications for theory and research. American Psychologist, 65, 237-251. doi:10.1037/a0019330.

Schwartz, S. J., Vignoles, V. L., Brown, R., \& Zagefka, H. (2014). The identity dynamics of acculturation and multiculturalism: Situating acculturation in context. In V. Benet-Martínez \& Y. Y. Hong (Eds.), Oxford handbook of multicultural identity (pp. 57-95). Oxford, UK: Oxford University Press.
Schwartz, S. J., Weisskirch, R. S., Zamboanga, B. L., Castillo, L. G., Ham, L. S., Huynh, Q.-L., . . . Cano, M. A. (2011). Dimensions of acculturation: Associations with health risk behaviors among college students from immigrant families. Journal of Counseling Psychology, 58, 27-41. doi:10.1037/a0021356

Schwartz, S. J., \& Zamboanga, B. L. (2008). Testing Berry's model of acculturation: A confirmatory latent class approach. Cultural Diversity and Ethnic Minority Psychology, 14, 275-285. doi:10.1037/a0012818

Schwartz, S. J., Zamboanga, B. L., \& Jarvis, L. H. (2007). Ethnic identity and acculturation in Hispanic early adolescents: Mediated relationships to academic grades, prosocial behaviors, and externalizing symptoms. Cultural Diversity and Ethnic Minority Psychology, 13, 364-373. doi:10.1037/1099-9809.13.4.364

Sessa, F. M., Avenevoli, S., Steinberg, L., \& Morris, A. S. (2001). Correspondence among informants on parenting: Preschool children, mothers, and observers. Journal of Family Psychology, 15, 53-68. doi:10.1037/0893-3200.15.1.53

Smith, P. B., Fischer, R., Vignoles, V. L., \& Bond, M. H. (2013). Understanding social psychology across cultures: Engaging with others in a changing world. London, UK: Sage.

Smokowski, P. R., \& Bacallao, M. L. (2007). Acculturation, internalizing mental health symptoms, and self-esteem: Cultural experiences of Latino Adolescents in North Carolina. Child Psychiatry and Human Development, 37, 273-292. doi:10.1007/s10578-006-0035-4

Smokowski, P. R., \& Bacallao, M. L. (2011). Becoming bicultural: Risk, resilience, and Latino youth. New York: New York University Press.

Smokowski, P. R., Rose, R. A., \& Bacallao, M. (2010). Influence of risk factors and cultural assets on Latino adolescents' trajectories of self-esteem and internalizing symptoms. Child Psychiatry and Human Development, 41, 133-155. doi:10.1007/s10578-009-0157-6

Steiner, N. (2009). International migration and citizenship today. New York, NY: Routledge.

Stepick, A., Grenier, G., Castro, M., \& Dunn, M. (2003). This land is our land: Immigrants and power in Miami. Berkeley: University of California Press.

Suárez-Orozco, C., \& Suárez-Orozco, M. M. (2001). Children of immigration. Cambridge, MA: Harvard University Press.

Suárez-Orozco, C., Suárez-Orozco, M. M., \& Todorova, I. L. G. (2008). Learning a new land. Cambridge, MA: Harvard University Press.

Szapocznik, J., Kurtines, W. M., \& Fernandez, T. (1980). Bicultural involvement and adjustment in HispanicAmerican youths. International Journal of Intercultural Relations, 4, 353-365.

Szapocznik, J., Rio, A., Perez-Vidal, A., Kurtines, W. M., Hervis, O. E., \& Santisteban, D. (1986). Bicultural effectiveness training (BET): An intervention modality for families experiencing intergenerational/intercultural conflict. Hispanic Journal of Behavioral Sciences, 6, 303330. doi:10.1177/07399863860084001 
Todorova, I. L. G., Falcón, L. M., Lincoln, A. K., \& Price, L. (2010). Perceived discrimination, psychological distress, and health. Sociology of Health and Illness, 32, 843-861. doi:10.1111/j.1467-9566.2010.01257.x

Tolan, P. H., Gorman-Smith, D., Huesmann, L. R., \& Zelli, A. (1997). Assessment of family relationship characteristics: A measure to explain risk for antisocial behavior and depression among urban youth. Psychological Assessment, 9, 212-223. doi:10.1037/1040-3590.9.3.212

Triandis, H. C. (1995). Individualism and collectivism. Boulder, CO: Westview.

Triandis, H. C., \& Gelfand, M. J. (1998). Converging measurement of horizontal and vertical individualism and collectivism. Journal of Personality and Social Psychology, 74, 118-128. doi:10.1037/0022-3514.74.1.118

Tropp, L. R., Erkut, S., García Coll, C., Alarcón, O., \& Vázquez García, H. A. (1999). Psychological acculturation: Development of a new measure for Puerto Ricans on the U.S. mainland. Educational and Psychological Measurement, 59, 351-367. doi:10.1177/00131649921 969794

Turner, C. F., Ku, L., Rogers, S. M., Lindberg, L. B., Pleck, J. H., \& Sonsenstein, L. H. (1998). Adolescent sexual behavior, drug use, and violence: Increased reporting with computer survey technology. Science, 280, 867873. doi:10.1126/science.280.5365.867
Umaña-Taylor, A. J., Gonzales-Backen, M. A., \& Guimond, A. B. (2009). Latino adolescents' ethnic identity: Is there a developmental progression and does growth in ethnic identity predict growth in self-esteem? Child Development, 80, 391-405. doi:10.1111/j.1467-8624.2009. 01267.x.

Umaña-Taylor, A. J., Quintana, S. M., Lee, R. M., Cross, W. E., Jr., Rivas-Drake, D., Schwartz, S. J., . . E Ethnic and Racial Identity in the 21st Century Study Group. (2014). Ethnic and racial identity revisited: An integrated conceptualization. Child Development, 85, 21-39. doi:10.1111/cdev.12196

Unger, J. B., Ritt-Olson, A., Soto, D. W., \& BaezcondeGarbanati, L. (2009). Parent-child acculturation discrepancies as a risk factor for substance use among Hispanic adolescents in Southern California. Journal of Immigrant and Minority Health, 11, 149-157. doi:10. 1007/s10903-007-9083-5

Walters, N. P., \& Trevelyan, E. N. (2011). The newly arrived foreign born population in the United States: 2010 (Census Brief ACSBR/10-16). Washington, DC: U.S. Census Bureau.

West, S. G., Taylor, A. B., \& Wu, W. (2012). Model fit and model selection in structural equation modeling. In $\mathrm{R}$. H. Hoyle (Ed.), Handbook of structural equation modeling (pp. 209-231). New York, NY: Guilford Press. 